\title{
Optical and photoemission investigation of structural and magnetic transitions in the iron-based superconductor $\mathrm{Sr}_{0.67} \mathrm{Na}_{0.33} \mathrm{Fe}_{2} \mathrm{As}_{2}$
}

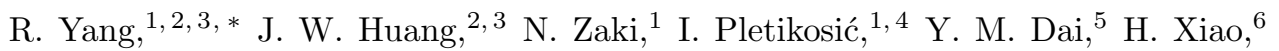

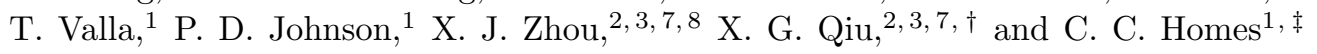 \\ ${ }^{1}$ Condensed Matter Physics and Materials Science Division, \\ Brookhaven National Laboratory, Upton, New York 11973, USA \\ ${ }^{2}$ Beijing National Laboratory for Condensed Matter Physics, \\ Institute of Physics, Chinese Academy of Sciences, Beijing 100190, China \\ ${ }^{3}$ School of Physical Sciences, University of Chinese Academy of Sciences, Beijing 100049, China \\ ${ }^{4}$ Department of Physics, Princeton University, Princeton, NJ 08544, USA \\ ${ }^{5}$ Center for Superconducting Physics and Materials, \\ National Laboratory of Solid State Microstructures and Department of Physics, Nanjing University, Nanjing 210093, China \\ ${ }^{6}$ Center for High Pressure Science and Technology Advanced Research, Beijing 100094, China \\ ${ }^{7}$ Songshan Lake Materials Laboratory, Dongguan 523808, China \\ ${ }^{8}$ Beijing Academy of Quantum Information Science, Beijing 100193, China
}

(Dated: June 25, 2021)

\begin{abstract}
We report the temperature dependent optical conductivity and angle-resolved photoemission spectroscopy (ARPES) studies of the multiband iron-based superconductor $\mathrm{Sr}_{0.67} \mathrm{Na}_{0.33} \mathrm{Fe}_{2} \mathrm{As}_{2}$. Measurements were made in the high-temperature tetragonal paramagnetic phase; below the structural and magnetic transitions at $T_{\mathrm{N}} \simeq 125 \mathrm{~K}$ in the orthorhombic spin-density-wave (SDW)-like phase, and $T_{r} \simeq 42 \mathrm{~K}$ in the reentrant tetragonal double- $\mathbf{Q}$ magnetic phase where both charge and SDW order exist; and below the superconducting transition at $T_{c} \simeq 10 \mathrm{~K}$. The free-carrier component in the optical conductivity is described by two Drude contributions; one strong and broad, the other weak and narrow. The broad Drude component decreases dramatically below $T_{\mathrm{N}}$ and $T_{r}$, with much of its strength being transferred to a bound excitation in the mid-infrared, while the narrow Drude component shows no anomalies at either of the transitions, actually increasing in strength at low temperature while narrowing dramatically. The behavior of an infrared-active mode suggests zone-folding below $T_{r}$. Below $T_{c}$ the dramatic decrease in the low-frequency optical conductivity signals the formation of a superconducting energy gap. ARPES reveals hole-like bands at the center of the Brillouin zone (BZ), with both electron- and hole-like bands at the corners. Below $T_{\mathrm{N}}$, the hole pockets at the center of the BZ decrease in size, consistent with the behavior of the broad Drude component; while below $T_{r}$ the electron-like bands shift and split, giving rise to a low-energy excitation in the optical conductivity at $\simeq 20 \mathrm{meV}$. The $C_{2}$ and $C_{4}$ magnetic states, with resulting spin-density-wave and charge-SDW order, respectively, lead to a significant reconstruction of the Fermi surface that has profound implications for the transport originating from the electron and hole pockets, but appears to have relatively little impact on the superconductivity in this material.
\end{abstract}

PACS numbers: 72.15.-v, 74.70.-b, 78.20.-e

\section{INTRODUCTION}

The discovery of iron-based superconductors prompted an intensive investigation in the hope of identifying new compounds with high superconducting critical temperatures $\left(T_{c}\right.$ 's) [1 4]. In many of the iron-based materials, superconductivity emerges with the suppression of antiferromagnetic (AFM) order, suggesting that the pairing mechanism is related to the magnetism. Indeed, the ironbased materials display a variety of magnetically-ordered ground states [5] 9] that may either compete with or foster the emergence of superconductivity.

One class of materials, $A e \mathrm{Fe}_{2} \mathrm{As}_{2}$, where $A e=\mathrm{Ba}, \mathrm{Ca}$

\footnotetext{
* Present address: Laboratorium für Festkörperphysik, ETH Zürich, CH-8093 Züich, Switzerland

$\dagger$ xgqiu@iphy.ac.cn

$\ddagger$ homes@bnl.gov
}

Typeset by REVTEX or Sr (the so-called " 122 " materials), is particularly useful as superconductivity may be induced through a variety of chemical substitutions [10 20, as well as through the application of pressure $21-24$. The phase diagram of $\mathrm{Sr}_{1-x} \mathrm{Na}_{x} \mathrm{Fe}_{2} \mathrm{As}_{2}$ has a number of interesting features. At room temperature, the parent compound $\mathrm{SrFe}_{2} \mathrm{As}_{2}$ is a paramagnetic metal with a tetragonal $(14 / \mathrm{mmm})$ structure. The resistivity in the iron-arsenic planes decreases with temperature until it drops anomalously as the material undergoes a magnetic transition at $T_{\mathrm{N}} \simeq 195 \mathrm{~K}$ to a spin-density-wave (SDW)-like AFM ground state that is also accompanied by a structural transition to an orthorhombic $(F m m m)$ phase [25 30]. The crystals are heavily twinned in the orthorhombic phase; however, the application of uniaxial stress along the (110) direction of the tetragonal unit cell results in a nearly twin-free sample 31, 32. The magnetic order may be described as AFM stripes, where the iron spins are aligned antiferromagnetically along the $a$ axis and ferromagnetically along 


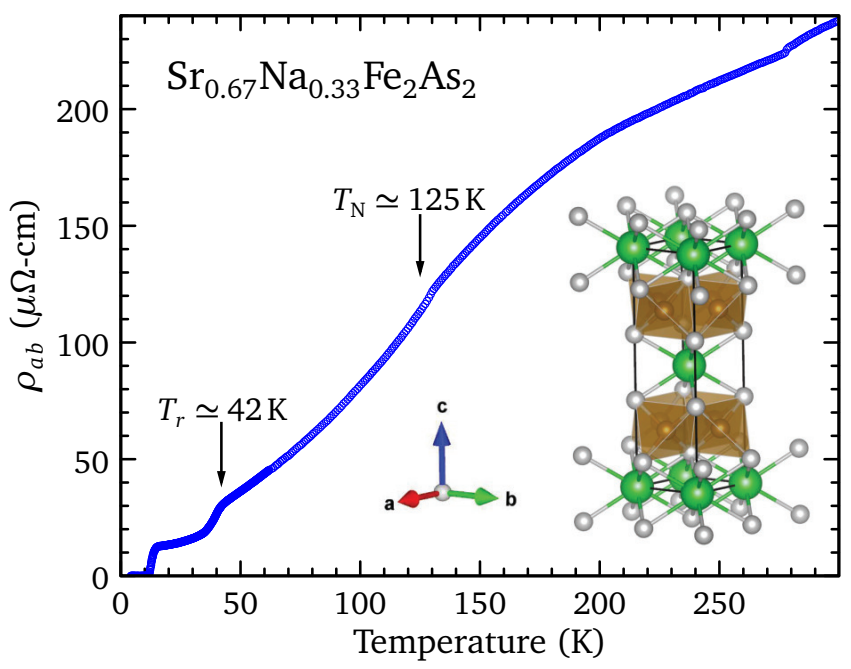

Figure 1. The temperature dependence of the in-plane resistivity for $\mathrm{Sr}_{0.67} \mathrm{Na}_{0.33} \mathrm{Fe}_{2} \mathrm{As}_{2}$ with inflection points at $T_{\mathrm{N}} \simeq$ $125 \mathrm{~K}$ and $T_{r} \simeq 42 \mathrm{~K}$; the resistivity at room temperature has been adjusted to match the optical conductivity in the zero-frequency limit. Inset: The generic unit cell in the hightemperature tetragonal phase for the 122 materials.

the $b$ axis [33, 34]; this is also referred to as the magnetic $C_{2}$ phase due to its twofold rotation symmetry. As the sodium content increases, the magnetic and structural transition temperatures decrease until both disappear at $x \simeq 0.48$; superconductivity appears well before this point at $x \simeq 0.2$, and reaches a maximum of $T_{c} \simeq 37 \mathrm{~K}$ for $x \simeq 0.5-0.6$. Between $0.29<x<0.42$, an additional magnetic and structural transition occurs below $T_{\mathrm{N}}$ at $T_{r}$; the tetragonal $(I 4 / \mathrm{mmm})$ phase reemerges, forming a dome which lies completely within the AFM region. This phase appears to be a common element in the hole-doped 122 materials 35-45; however, in $\mathrm{Sr}_{1-x} \mathrm{Na}_{x} \mathrm{Fe}_{2} \mathrm{As}_{2}$ the dome is more robust and occurs over a wider doping range at temperatures up to $T_{r} \simeq 65 \mathrm{~K}$ [39, 40], which is higher than has been observed in other compounds. This magnetic order is described as the collinear superposition of two itinerant SDW's with nesting wavevector $\mathbf{Q}$, leading to a double-Q SDW [4, 45] in which half the iron sites are nonmagnetic, and half have twice the moment measured in the orthorhombic AFM phase, oriented along the $c$ axis [46, 47]; this is referred to as the magnetic $C_{4}$ phase because of its fourfold rotational invariance. This magnetic state is accompanied by a charge-density wave (CDW) with the charge coupling to the square of the magnetization, resulting in a charge-SDW (CSDW) [48].

In this work, the complex optical properties and angle-resolved photoemission spectroscopy (ARPES), of $\mathrm{Sr}_{0.67} \mathrm{Na}_{0.33} \mathrm{Fe}_{2} \mathrm{As}_{2}$ have been investigated in the hightemperature tetragonal phase, as well as the magnetic $C_{2}$ and $C_{4}$ phases. The value of $x \simeq 0.33$ used in the current study is slightly below the optimal value of $x \simeq 0.37$ that bisects the $C_{4}$ dome in the $\mathrm{Sr}_{1-x} \mathrm{Na}_{x} \mathrm{Fe}_{2} \mathrm{As}_{2}$ phase diagram [39]. Based on transport studies, $T_{\mathrm{N}} \simeq 125 \mathrm{~K}$,
$T_{r} \simeq 42 \mathrm{~K}$, and $T_{c} \simeq 10 \mathrm{~K}$. In the high temperature tetragonal paramagnetic state, the optical response of the free-carriers is described by two Drude terms (Sec. IIIA); one strong and broad (large scattering rate), and the other weak and narrower (smaller scattering rate); as the temperature is reduced, the strength of the Drude terms show relatively little temperature dependence, while the scattering rates slowly decrease. Below $T_{\mathrm{N}}$, the Fermi surface reconstruction driven by the structural and magnetic transitions causes both the strength and the scattering rate for the broad Drude term to decrease dramatically; the missing spectral weight (the area under the conductivity curve) associated with the free carriers is transferred to a peak that emerges in the mid-infrared. The narrow Drude term actually increases slightly in strength below $T_{\mathrm{N}}$ while narrowing. Below $T_{r}$, in the magnetic $C_{4}$ phase, the broad Drude term again narrows and decreases in strength; while the strength of the narrow term does not appear to change, its scattering rate decreases dramatically. Based on the behavior of an infrared-active lattice mode, the presence of CSDW order likely results in the formation of a supercell resulting in zone folding, leading to a further reconstruction of the Fermi surface; while spectral weight is again transferred from the broad Drude to the midinfrared peak, a new low-energy peak emerges at $\simeq 20 \mathrm{meV}$. Below $T_{c}$, there is a dramatic decrease in the low-frequency conductivity, signalling the formation of a superconducting energy gap. ARPES reveals several large hole pockets at the center of the Brillouin zone above $T_{\mathrm{N}}$, one of which shifts below the Fermi level below $T_{\mathrm{N}}$ in the $C_{2}$ magnetic phase, a trend which continues below $T_{r}$, suggesting that these bands may be related to the broad Drude response. At the corners of the Brillouin zone, there are both hole- and electron-like bands. Below $T_{\mathrm{N}}$ and $T_{r}$, several of these bands appear to split and shift, but it is not clear if there are any significant changes to the size of the associated Fermi surfaces, suggesting that some of these carriers may be related to the narrow Drude term; below $T_{r}$ the band splitting is likely responsible for the emergence of the low-energy peak. The structural and magnetic transitions from which the $C_{2}$ (SDW) and $C_{4}$ (double-Q SDW) phases emerge result in a Fermi surface reconstruction that has profound effects on the optical conductivity and electronic structure; however, the superfluid stiffness appears to be more or less unaffected by the CSDW order.

\section{EXPERIMENT}

High-quality single crystals of $\mathrm{Sr}_{0.67} \mathrm{Na}_{0.33} \mathrm{Fe}_{2} \mathrm{As}_{2}$ with good cleavage planes (001) were synthesized using a selfflux technique [39, 49]. The temperature dependence of the in-plane resistivity, shown in Fig. 1. was measured using a standard four-probe configuration using a Quantum Design physical property measurement system; the unit cell for the high-temperature tetragonal phase is 

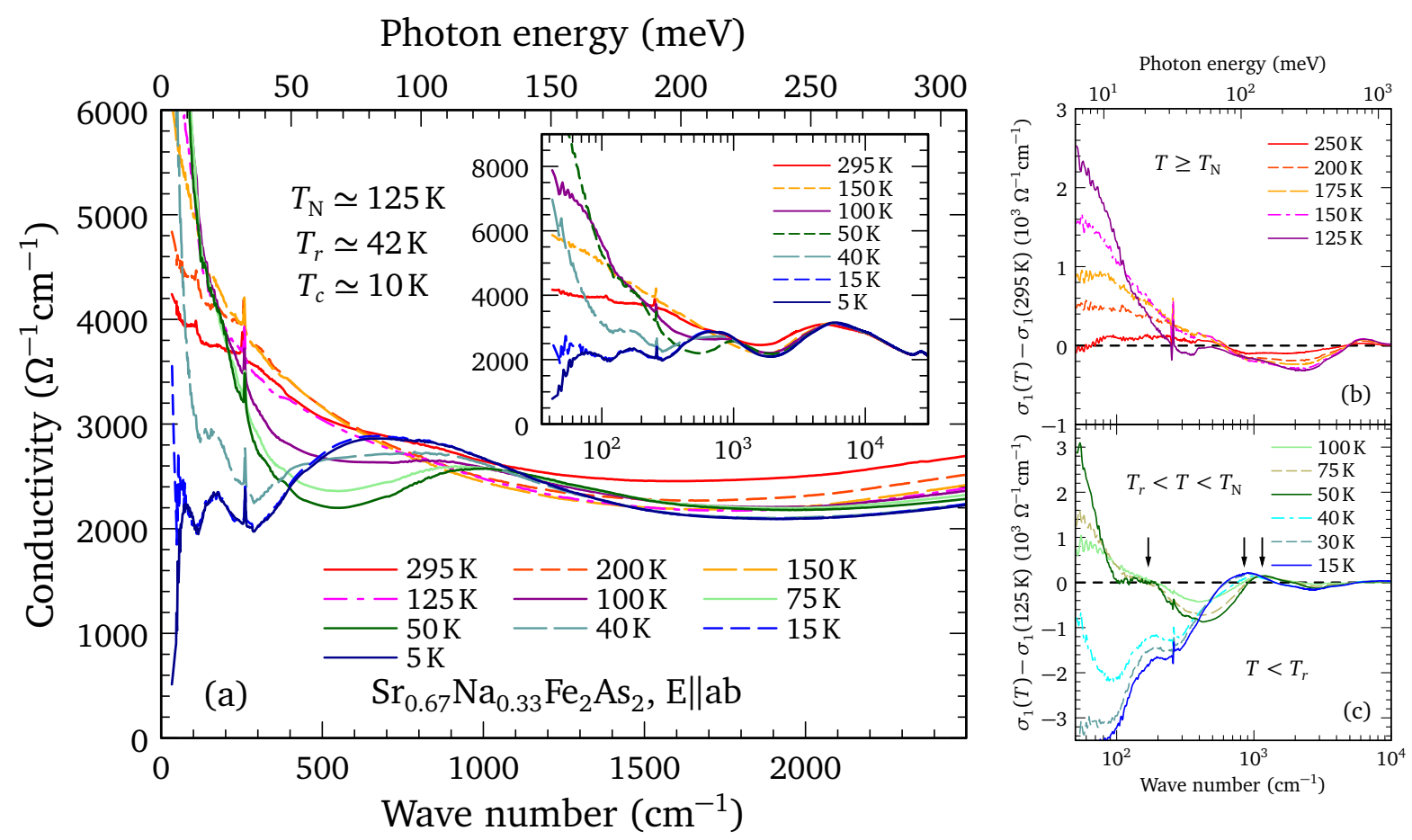

Figure 2. (a) The temperature dependence of the real part of the optical conductivity of $\mathrm{Sr}_{0.67} \mathrm{Na}_{0.33} \mathrm{Fe}_{2} \mathrm{As}_{2}$ in the infrared region for light polarized in the $\mathrm{Fe}-$ As planes. Inset: the conductivity over a wide spectral range at several temperatures. (b) The $\sigma_{1}(\omega, T)-\sigma_{1}(\omega, 295 \mathrm{~K})$ difference plot for $T \geq T_{\mathrm{N}}$ over a wide spectral range showing the narrowing of the free-carrier response and the transfer of spectral weight from high to low frequency. (c) The $\sigma_{1}(\omega, T)-\sigma_{1}(\omega, 125 \mathrm{~K})$ difference plot. Int the $T_{r}<T<T_{\mathrm{N}}$ region the free-carrier response continues to narrow and a peak emerges in the mid-infrared region; for $T<T_{r}$, the low-frequency conductivity is further suppressed, the mid-infrared peak shifts to low energy, and a prominent peak is observed at $\simeq 170 \mathrm{~cm}^{-1}$ (arrows).

shown in the inset. The resistivity decreases gradually with temperature, showing a weak inflection point at $T_{\mathrm{N}} \simeq 125 \mathrm{~K}$ with a more pronounced decrease in the resistivity at $T_{r} \simeq 42 \mathrm{~K}$; the resistivity goes to zero below the superconducting transition at $T_{c} \simeq 10 \mathrm{~K}$. The reflectance from freshly-cleaved surfaces has been measured at a near-normal angle of incidence over a wide temperature $(\simeq 5$ to $300 \mathrm{~K})$ and frequency range $(\simeq 2 \mathrm{meV}$ to about $5 \mathrm{eV}$ ) with Bruker IFS $113 \mathrm{v}$ and Vertex $80 \mathrm{v}$ Fourier transform spectrometers for light polarized in the $a-b$ planes using an in situ evaporation technique [50]. The complex optical properties have been determined from a Kramers-Kronig analysis of the reflectivity. The reflectivity is shown in supplementary Fig. S1; the details of the Kramers-Kronig analysis are described in the Supplementary Material [51. Temperature dependent ARPES measurements have been performed to track the evolution of the electron and hole pockets in the various phases. Measurements at BNL, which focused on the electronic structure near the center of the Brillouin zone, were performed using $21.2 \mathrm{eV}$ light from a monochromator-filtered He I source (Omicron VUV5k) and a Scienta SES-R4000 electron spectrometer; emitted electrons were collected along the direction perpendicular to the light-surface mirror plane. Samples were cleaved at low temperature and measured in an ultrahigh vacuum with a base pressure better than $5 \times 10^{-10}$ mbar. Measurements at the National Laboratory for Superconductivity, Institute of Physics, Chinese Academy of Sciences, were performed using a $21.2 \mathrm{eV}$ helium discharge lamp and a Scienta DA30L electron spectrometer. The latter's overall energy resolution was $10 \mathrm{meV}$ for Fermi surface mapping and $4 \mathrm{meV}$ for the cuts; the angular resolution was $\sim 0.1^{\circ}$. All the samples were cleaved at low temperature and measured in an ultrahigh vacuum with a base pressure better than $5 \times 10^{-11}$ mbar. Note that because uniaxial strain is not applied to the samples below $T_{\mathrm{N}}$, they will be heavily twinned, thus the optical and ARPES results represent an average of the $a$ and $b$ axis response in the magnetic $C_{2}$ phase.

\section{RESULTS AND DISCUSSION}

\section{A. Optical properties}

The temperature dependence of the real part of the inplane optical conductivity $\left[\sigma_{1}(\omega)\right]$ of $\mathrm{Sr}_{0.67} \mathrm{Na}_{0.33} \mathrm{Fe}_{2} \mathrm{As}_{2}$ is shown in the infrared region in Fig. 2(a) (an additional plot of the optical conductivity is shown in supplemen- 

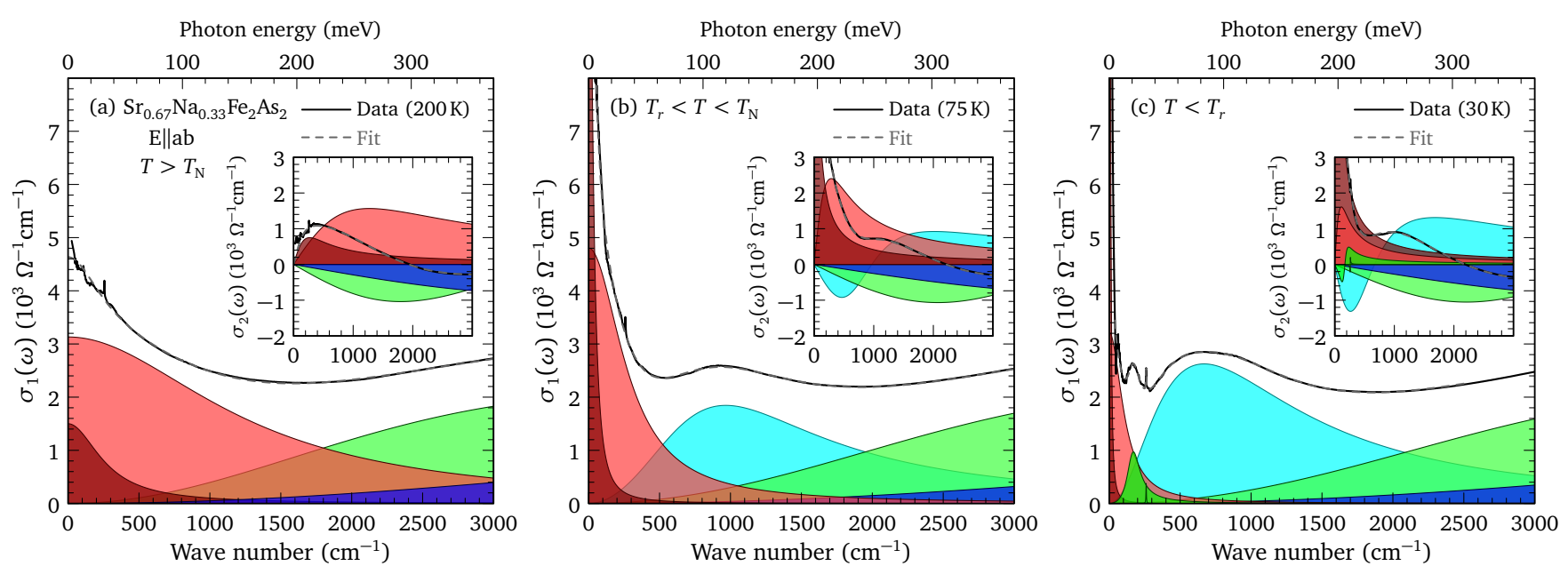

Figure 3. The Drude-Lorentz model fits to the real and imaginary (inset) parts of the in-plane optical conductivity of $\mathrm{Sr}_{0.67} \mathrm{Na}_{0.33} \mathrm{Fe}_{2} \mathrm{As}_{2}$ decomposed into the narrow (D1) and broad (D2) Drude components, as well as several bound excitations (a) above $T_{\mathrm{N}}$ at $200 \mathrm{~K}$, (b) below $T_{\mathrm{N}}$ at $75 \mathrm{~K}$ showing the narrowing of the Drude features and the emergence of a peak at $\simeq 950 \mathrm{~cm}^{-1}$, and (c) below $T_{r}$ at $30 \mathrm{~K}$, showing further narrowing and peaks at $\simeq 170$ and $700 \mathrm{~cm}^{-1}$.

tary Fig. S2). The character of the conductivity changes dramatically through the structural and magnetic transitions, which can be characterized by four distinct regions: (i) $T>T_{\mathrm{N}}$; (ii) $T_{r}<T<T_{\mathrm{N}}$; (iii) $T<T_{r}$, and below the superconducting transition (iv) $T<T_{c}$. The changes to the nature of the conductivity are shown as the difference plots $\sigma_{1}(\omega, T)-\sigma_{1}(\omega, 295 \mathrm{~K})$, and $\sigma_{1}(\omega, T)-\sigma_{1}(\omega, 125 \mathrm{~K})$, shown in Figs. 2(b) and 2(c), respectively.

At room temperature, the free-carrier response appears Drude-like (a Lorentzian centered at zero frequency with a scattering rate defined as the full width at half maximum), giving way to a flat response at higher frequencies, until the first interband transitions are encountered at about $1 \mathrm{eV}$. As the temperature is reduced, the scattering rate decreases and there is a slight reduction of the conductivity in the mid-infrared region as spectral weight is transferred from high to low frequency, which leads to an increase at low frequency and a decrease at high frequency in the difference spectra in Fig. 2(b). Below $T_{\mathrm{N}}$ in the $C_{2}$ phase, the free-carrier response narrows dramatically and a peak-like structure emerges at about $950 \mathrm{~cm}^{-1}$, somewhat lower than a similar feature that was observed below $T_{\mathrm{N}}$ at $\simeq 1400 \mathrm{~cm}^{-1}$ in the parent compound $\mathrm{SrFe}_{2} \mathrm{As}_{2}$ [52. This is illustrated by the upper three curves in Fig.2(c) that show the continuing increase in the low-frequency conductivity, as well as the emergence of a peak in the mid-infrared region. Interestingly, below $\simeq 75 \mathrm{~K}$, a low-energy peak at $\simeq 170 \mathrm{~cm}^{-1}$ begins to emerge. This behavior continues until $T \leq T_{r}$, at which point the Drude-like response becomes extremely narrow in the $C_{4}$ phase, illustrated by the dramatic suppression of the low-frequency conductivity in the difference plot in Fig. 2(c), leaving clearly identifiable peaks at $\simeq 170$ and $700 \mathrm{~cm}^{-1}$. Below $T_{c} \simeq 10 \mathrm{~K}$, there is a depletion of the low-frequency conductivity with the emergence of a shoulder-like structure around $70 \mathrm{~cm}^{-1}$ that signals the formation of a superconducting energy gap (supplementary Fig. S2).

The sharp feature observed in the conductivity at $\simeq 260 \mathrm{~cm}^{-1}$ is attributed to a normally infrared-active lattice vibration in the iron-arsenic planes; while this mode increases in frequency with decreasing temperature, it does not display the anomalous increase in oscillator strength below $T_{\mathrm{N}}$ that was observed in the parent compound [53. However, below $T_{r}$ there is evidence for a new satellite mode appearing at $\simeq 282 \mathrm{~cm}^{-1}$ (supplementary Fig. S3); a similar feature has also been observed in the $C_{4}$ phase of $\mathrm{Ba}_{1-x} \mathrm{~K}_{x} \mathrm{Fe}_{2} \mathrm{As}_{2}$ and is attributed to Brillouin-zone folding due to the formation of a supercell in the CSDW phase [54].

Previous optical studies of the iron-arsenic materials recognized that these are multiband materials with hole and electron pockets at the center and corners of the Brillouin zone [55, [56]; a minimal description consists of two electronic subsystems using the so-called two-Drude model [57]. The complex dielectric function $\tilde{\epsilon}=\epsilon_{1}+i \epsilon_{2}$ can be written as,

$$
\tilde{\epsilon}(\omega)=\epsilon_{\infty}-\sum_{j=1}^{2} \frac{\omega_{p, D ; j}^{2}}{\omega^{2}+i \omega / \tau_{D, j}}+\sum_{k} \frac{\Omega_{k}^{2}}{\omega_{k}^{2}-\omega^{2}-i \omega \gamma_{k}},
$$

where $\epsilon_{\infty}$ is the real part at high frequency. In the first sum, $\omega_{p, D ; j}^{2}=4 \pi n_{j} e^{2} / m_{j}^{*}$ and $1 / \tau_{D, j}$ are the square of the plasma frequency and scattering rate for the delocalized (Drude) carriers in the $j$ th band, respectively, and $n_{j}$ and $m_{j}^{*}$ are the carrier concentration and effective mass. In the second summation, $\omega_{k}, \gamma_{k}$ and $\Omega_{k}$ are the position, width, and strength of the $k$ th vibration or bound excitation. The complex conductivity is $\tilde{\sigma}(\omega)=\sigma_{1}+i \sigma_{2}=-2 \pi i \omega\left[\tilde{\epsilon}(\omega)-\epsilon_{\infty}\right] / Z_{0}$ (in units of $\left.\Omega^{-1} \mathrm{~cm}^{-1}\right) ; Z_{0} \simeq 377 \Omega$ is the impedance of free space. The model is fit to the real and imaginary parts of the 

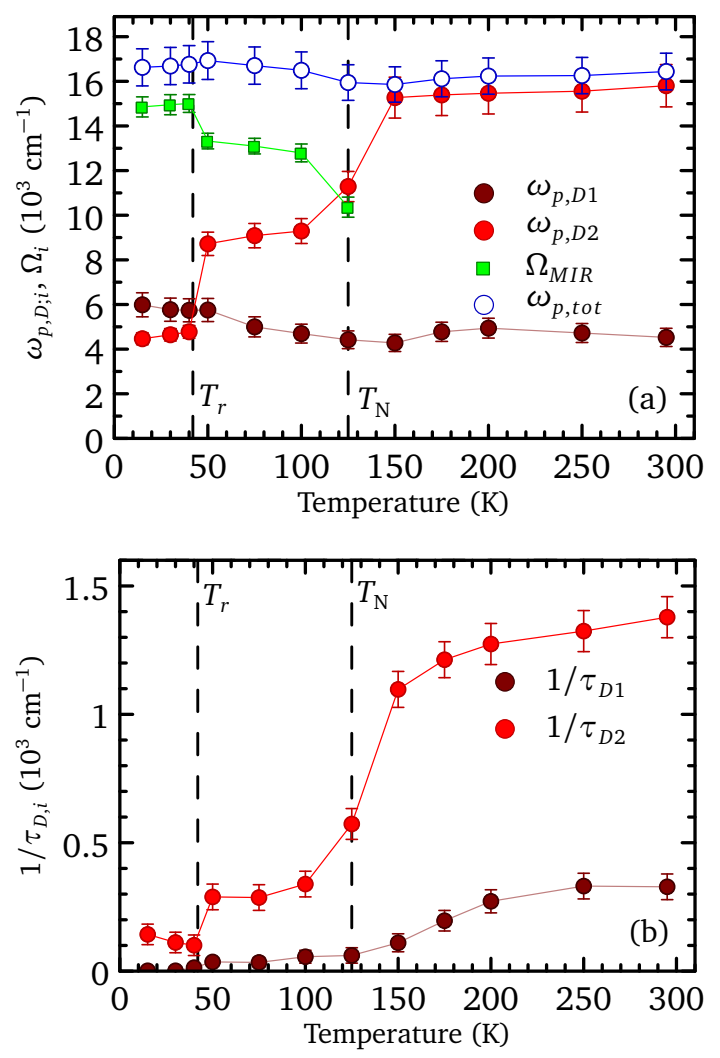

Figure 4. (a) The temperature dependence of the plasma frequencies of the narrow (D1) and broad (D2) Drude components, the oscillator strength of the mid-infrared peak $\left(\Omega_{\mathrm{MIR}}\right.$, and the total when these three components are added in quadrature $\left(\omega_{p, t o t}\right)$, for $\mathrm{Sr}_{0.67} \mathrm{Na}_{0.33} \mathrm{Fe}_{2} \mathrm{As}_{2}$. (b) The temperature dependence of the scattering rates of the narrow and broad Drude components.

optical conductivity simultaneously using a non-linear least-squares technique. The results of the fits are shown in Figs. 3(a), 3(b), and 3(c) at $200 \mathrm{~K}\left(T>T_{\mathrm{N}}\right), 75 \mathrm{~K}$ $\left(T_{r}<T<T_{\mathrm{N}}\right)$, and $30 \mathrm{~K}\left(T<T_{r}\right)$, respectively; the combined response has been decomposed into individual Drude and Lorentz components. In agreement with previous studies on the iron-based materials, the complex conductivity can be described by two Drude terms, one weak and narrow (D1), the other strong and broad (D2), as well as several Lorentzian oscillators. The temperature dependence of the plasma frequencies, the D1 and D2 components, as well as the strength of the midinfrared (MIR) peak, are shown in Fig. 4(a); the temperature dependence of the scattering rates for the two Drude components is shown in Fig. 4(b).

$$
\text { 1. } T>T_{\mathrm{N}}
$$

At room temperature, the plasma frequencies for the narrow and broad Drude terms, $\omega_{p, D 1} \simeq 4400 \mathrm{~cm}^{-1}$ and $\omega_{p, D 2} \simeq 15800 \mathrm{~cm}^{-1}$, respectively, are slightly less than those of the undoped parent compound $\mathrm{SrFe}_{2} \mathrm{As}_{2}$ $\left(\omega_{p, D 1} \simeq 5200 \mathrm{~cm}^{-1}\right.$ and $\left.\omega_{p, D 2} \simeq 17700 \mathrm{~cm}^{-1}\right)$; however, the scattering rates of $1 / \tau_{D 1} \simeq 330 \mathrm{~cm}^{-1}$ and $1 / \tau_{D 2} \simeq 1400 \mathrm{~cm}^{-1}$ are noticeably lower than the values of $1 / \tau_{D 1} \simeq 470 \mathrm{~cm}^{-1}$ and $1 / \tau_{D 2} \simeq 2330 \mathrm{~cm}^{-1}$ observed in the undoped material 52. This is somewhat surprising considering that in this material the layers in between the $\mathrm{Fe}-\mathrm{As}$ sheets are disordered. While the plasma frequencies show little temperature dependence between room temperature and $T_{\mathrm{N}}$, the scattering rates for both Drude components decrease with temperature, with the narrow Drude decreasing from about $1 / \tau_{D 1} \simeq 330$ to about $60 \mathrm{~cm}^{-1}$, and the broad Drude decreasing from $1 / \tau_{D 2} \simeq 1400 \mathrm{~cm}^{-1}$ to about $1100 \mathrm{~cm}^{-1}$ just above $T_{\mathrm{N}}$.

$$
\text { 2. } T_{r}<T<T_{\mathrm{N}}
$$

Below $T_{\mathrm{N}}$ in the magnetic $C_{2}$ phase, the plasma frequency for the narrow Drude increases slightly from $\omega_{p, D 1} \simeq 4400$ to $\simeq 6000 \mathrm{~cm}^{-1}$, while the scattering rate continues to decrease to $1 / \tau_{D 1} \simeq 40 \mathrm{~cm}^{-1}$ just above $T_{r}$. The broad Drude displays much larger changes, with the plasma frequency decreasing from $\omega_{p, D 2} \simeq 15800$ to $9000 \mathrm{~cm}^{-1}$, which corresponds to a decrease in carrier concentration of nearly $65 \%\left(\omega_{p}^{2} \propto n / m^{*}\right)$; the scattering rate also drops dramatically from $1 / \tau_{D 2} \simeq 1100 \mathrm{~cm}^{-1}$ just above $T_{\mathrm{N}}$ to $300 \mathrm{~cm}^{-1}$ in the $T_{r}<T<T_{\mathrm{N}}$ region. The dramatic loss of spectral weight of the broad Drude term is accompanied by the emergence of a new peak in the MIR region with position $\omega_{\mathrm{MIR}} \simeq 950 \mathrm{~cm}^{-1}$, width $\gamma_{\mathrm{MIR}} \simeq 1550 \mathrm{~cm}^{-1}$, and strength $\Omega_{\mathrm{MIR}} \simeq 13000 \mathrm{~cm}^{-1}$ [Fig. 3(b)]; the missing weight from the free carriers is transferred into this bound excitation, and accordingly the total spectral weight is defined as $\omega_{p, t o t}^{2}=$ $\omega_{p, D 1}^{2}+\omega_{p, D 2}^{2}+\Omega_{\mathrm{MIR}}^{2}$, is constant, as shown in Fig. 4 (a). This behavior is similar to what was previously observed in the parent compound, and has been explained as the partial gapping of the pocket responsible for the broad Drude term due and the appearance of a low-energy interband transition [52, 58].

$$
\text { 3. } T<T_{r}
$$

As the temperature is reduced the system undergoes a further magnetic and structural transition at $T_{r} \simeq 42 \mathrm{~K}$ and enters the magnetic $C_{4}$ phase. Below $T_{r}$ the plasma frequency for the narrow Drude term appears to actually increase slightly; however, this is accompanied by a dramatic collapse of $1 / \tau_{D 1} \simeq 40 \mathrm{~cm}^{-1}$ just above $T_{r}$ to a value of $\simeq 2 \mathrm{~cm}^{-1}$ at $15 \mathrm{~K}$; this is nearly an order of magnitude smaller than what is observed in the parent compound [52. Consequently, the narrow Drude is no longer observable in $\sigma_{1}(\omega)$, leaving a relatively flat optical conductivity due to the broad Drude term and Lorentzian components; instead, its effects are determined from $\sigma_{2}(\omega)$ [shown in the inset of Fig. 3(c)]. 


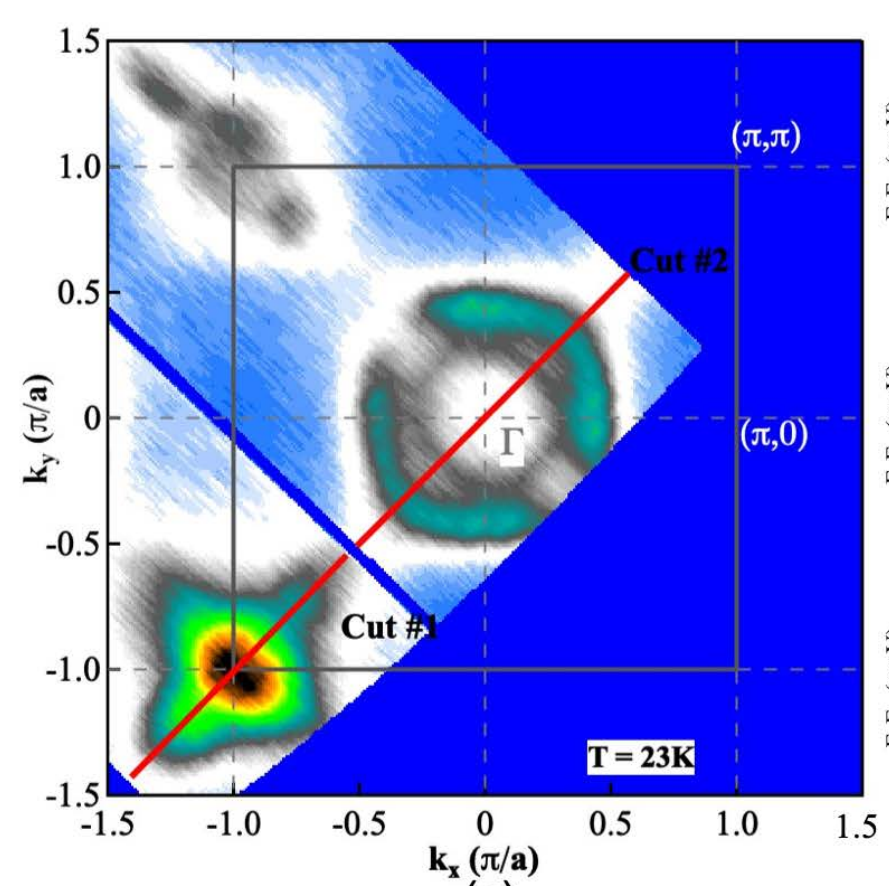

(a)

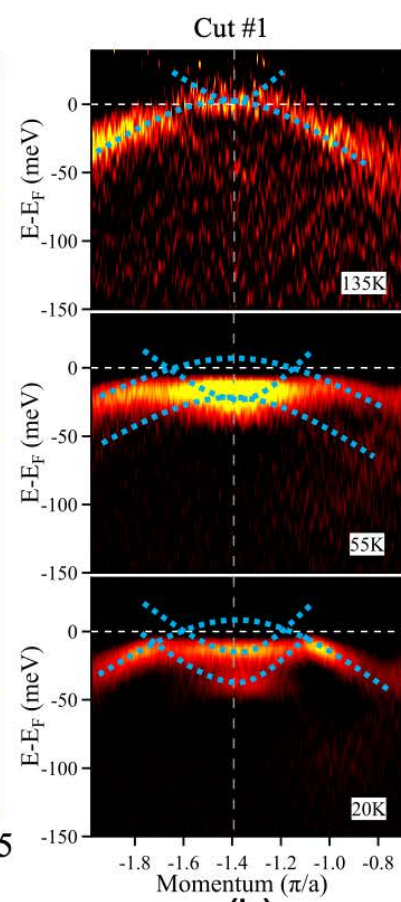

(b)

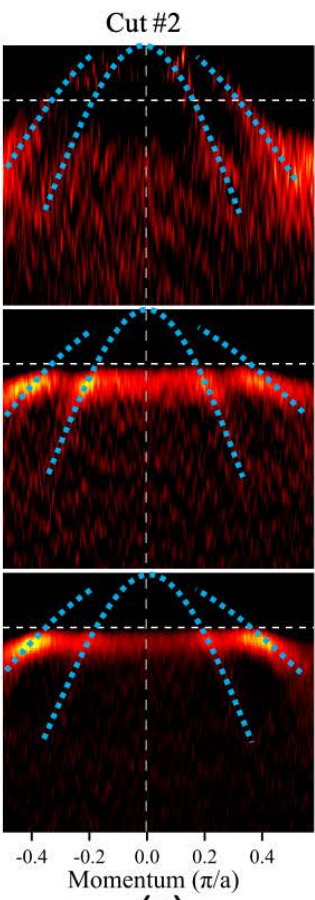

(c)

Figure 5. (a) Fermi surface mapping of $\mathrm{Sr}_{0.67} \mathrm{Na}_{0.33} \mathrm{Fe}_{2} \mathrm{As}_{2}$ in the $C_{4}$ magnetic phase at $23 \mathrm{~K}$ with the spectral weight integrated within a $\pm 10 \mathrm{meV}$ energy window with respect to the Fermi level, showing the hole-like pockets at the center $(\Gamma)$, and the electron-like pockets at the corner $(\mathrm{M})$ of the Brillouin zone. Several different cuts are shown along the $\Gamma \rightarrow \mathrm{M}$ path focus on the evolution of the hole and electron pockets. (b) The temperature dependence of the second derivative of the energy bands measured along the first cut around the $\mathrm{M}$ point at $(-\pi,-\pi)$ at $135 \mathrm{~K}\left(T>T_{\mathrm{N}}\right), 55 \mathrm{~K}\left(T_{r}<T<T_{\mathrm{N}}\right)$, and $20 \mathrm{~K}\left(T_{c}<T<T_{r}\right)$. (c) The temperature dependence of the second derivative of the energy bands measured along the second cut around the $\Gamma$ point at 135,55 , and $20 \mathrm{~K}$. The dotted lines are drawn as a guide to the eye.

The plasma frequency of the broad Drude term continues to decrease from $\omega_{p, D 2} \simeq 9000$ to about $4200 \mathrm{~cm}^{-1}$ at $15 \mathrm{~K}$, a further $80 \%$ reduction in the carrier concentration associated with this pocket, and over $90 \%$ from the room temperature value; this is comparable to what was observed in the parent compound for $T \ll T_{\mathrm{N}}$ 52. In addition, the scattering rate decreases from $1 / \tau_{D 2} \simeq 300 \mathrm{~cm}^{-1}$ at $T_{r}$ to $\simeq 120 \mathrm{~cm}^{-1}$ at $15 \mathrm{~K}$. At the same time, the peak at $\omega_{\mathrm{MIR}} \simeq 950 \mathrm{~cm}^{-1}$ shifts down to about $\simeq 650 \mathrm{~cm}^{-1}$; while the width decreases slightly to $\gamma_{\mathrm{MIR}} \simeq 1480 \mathrm{~cm}^{-1}$, the strength of this feature increases to $\Omega_{\mathrm{MIR}} \simeq 15400 \mathrm{~cm}^{-1}$. However, $\omega_{p, t o t}$ continues to be conserved, indicating that the loss of spectral weight associated with the free carriers in the broad Drude term has been transferred to this peak.

$$
\text { 4. } T<T_{c}
$$

Below $T_{c} \simeq 10 \mathrm{~K}$ there is a dramatic suppression of the low-frequency conductivity, signalling the formation of a superconducting energy gap [Fig. 2(a) and supplementary Fig. S2]. Although the low-frequency data is somewhat limited, a comparison of the optical conduc- tivity for $T \gtrsim T_{c}$ and $T \ll T_{c}$ allows the superfluid density, $\rho_{s}=\omega_{p s}^{2}$, where $\omega_{p s}$ is the superconducting plasma frequency, to be determined from the missing spectral weight, calculated using the Ferrell-Glover-Tinkham (FGT) sum rule [59, 60. The FGT sum rule converges to $\omega_{p s} \simeq 5800 \pm 500 \mathrm{~cm}^{-1}$, which corresponds to a superconducting penetration depth of $\lambda \simeq 2700 \pm 300 \AA$ at $5 \mathrm{~K}$, comparable to the K-doped material [47; however, because the lowest temperature obtained was only $\simeq T_{c} / 2$, it is almost certain that $\omega_{p s}$ is underestimated. From Fig. 2(a) and supplementary Fig. S2, the characteristic energy scale for the superconducting energy gap is about $2 \Delta \simeq 50 \mathrm{~cm}^{-1}$. In the narrow Drude band, $1 / \tau_{D 1} \ll 2 \Delta$, placing this material in the clean limit; as a result, most of the weight in the condensate will come from this band. In the broad Drude band, $1 / \tau_{D 2}>2 \Delta$, placing this band in the dirty limit; consequently, only a small fraction of the weight in this band will collapse into the condensate. This is another example of a multiband iron-based superconductor that is simultaneously in both the clean and dirty limits [61. One of the interesting properties of this material is its relatively low resistivity just above $T_{c}, \rho_{a b} \simeq 20 \mu \Omega \mathrm{cm}$, or $\sigma_{d c} \simeq 5 \times 10^{4} \Omega^{-1} \mathrm{~cm}^{-1}$ [Fig 1 . These values place this material just below the univer- 

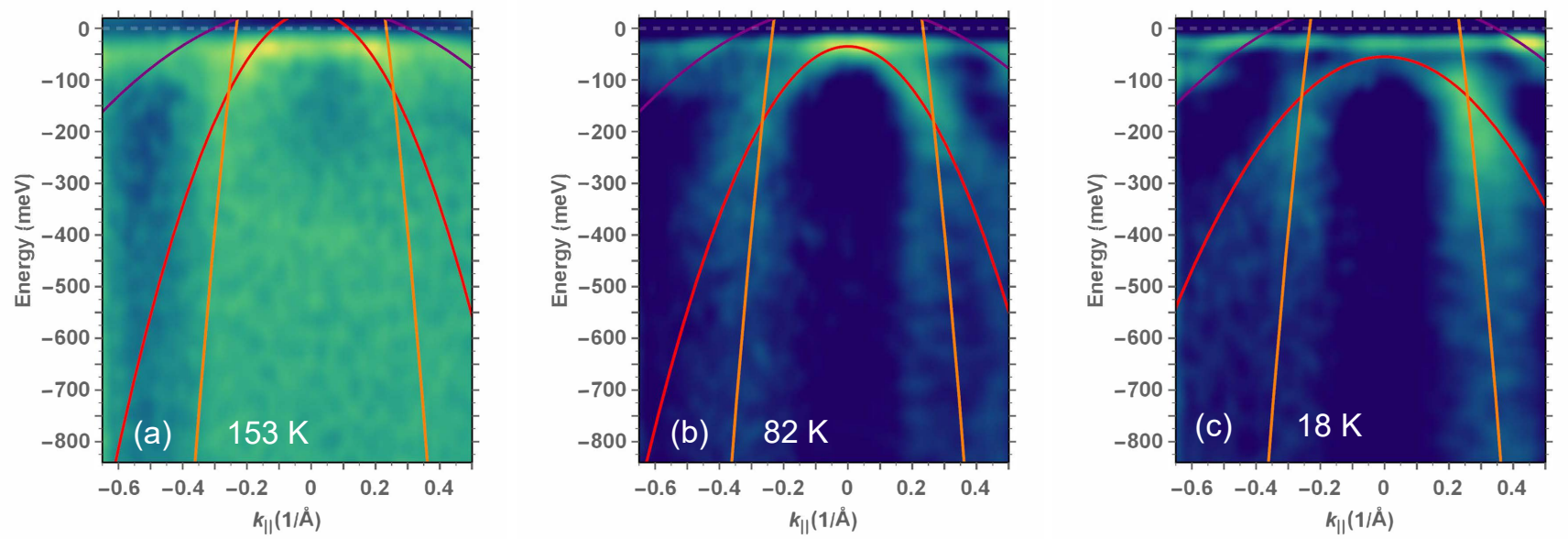

Figure 6. The temperature dependence of the second-derivative of the hole-like bands of $\mathrm{Sr}_{0.67} \mathrm{Na}_{0.33} \mathrm{Fe}_{2} \mathrm{As}_{2}$ around the $\Gamma$ point along the $\Gamma \rightarrow \mathrm{M}$ cut at: (a) above $T_{\mathrm{N}}$ at $153 \mathrm{~K},(\mathrm{~b})$ for $T_{r}<T<T_{\mathrm{N}}$ at $82 \mathrm{~K}$, and (c) below $T_{r}$ at $18 \mathrm{~K}$. At high temperature three hole-like bands may be resolved that cross $\epsilon_{F}$. Below $T_{\mathrm{N}}$ one of these bands shift to below the Fermi level; this trend continues below $T_{r}$ as the bands shift further below $\epsilon_{F}$. The lines are drawn as a guide to the eye.

sal scaling line $\rho_{s}\left(T \ll T_{c}\right) \propto \sigma_{d c}\left(T \gtrsim T_{c}\right) T_{c} 62$ 64, in close proximity to other doped " 122 " superconductors, as well as many cuprate materials [65].

\section{B. Low-energy peak}

The dramatic collapse of the scattering rate below $T_{r}$ of the narrow Drude allows a new low-energy peak at $\omega_{0} \simeq 170 \mathrm{~cm}^{-1}$, with width $\gamma_{0} \simeq 110 \mathrm{~cm}^{-1}$ and oscillator strength of $\Omega_{0} \simeq 2230 \mathrm{~cm}^{-1}$, to be observed [Figs. 2(a), 3(c), and supplementary Fig. S2]. This is close to where a peak was observed in $\left(\mathrm{CaFe}_{1-x} \mathrm{Pt}_{x} \mathrm{As}\right)_{10} \mathrm{Pt}_{3} \mathrm{As}_{8}$ for $x=$ 0.1 at $\simeq 120 \mathrm{~cm}^{-1}[66$; that feature was attributed to a localization process due to impurity scattering described by a classical generalization of the Drude model [67,

$$
\tilde{\sigma}(\omega)=\left(\frac{2 \pi}{Z_{0}}\right) \frac{\omega_{p}^{2} \tau}{(1-i \omega \tau)}\left[1+\frac{c}{(1-i \omega \tau)}\right]
$$

where $c$ is the persistence of velocity that is retained for a single collision. The scattering rate for the narrow Drude is far too small to yield a peak at the experimentally-observed position, while the broad Drude predicts a localization peak at $\simeq 120 \mathrm{~cm}^{-1}$, well below the experimentally-observed value of $\omega_{0} \simeq 170 \mathrm{~cm}^{-1} 68$. Thus, it is likely that the low-energy peak originates from a further reconstruction of the Fermi surface in the $C_{4}$ phase rather than any sort of localization process. Indeed, a remarkably similar peak has also been observed to emerge at $\simeq 150 \mathrm{~cm}^{-1}$ in the optical conductivity of underdoped $\mathrm{Ba}_{1-x} \mathrm{~K}_{x} \mathrm{Fe}_{2} \mathrm{As}_{2}$ at low temperature [69]; this feature may also be a related to the magnetic $C_{4}$ phase observed in that compound.

\section{ARPES}

A simple density functional theory calculation of $\mathrm{SrFe}_{2} \mathrm{As}_{2}$ in the paramagnetic high-temperature tetragonal phase reveals a familiar band structure consisting of three hole-like pockets at the center of the Brillouin zone $(\Gamma)$, and two electron-like pockets at the corners $(\mathrm{M})$; the orbital character is primarily Fe $d_{x z} / d_{y z}$ in nature (shown in supplementary Fig. S4, details of the calculation are discussed in the Supplementary Material.) The Fermi surface of $\mathrm{Sr}_{0.67} \mathrm{Na}_{0.33} \mathrm{Fe}_{2} \mathrm{As}_{2}$, with the spectral weight integrated within a $\pm 10 \mathrm{meV}$ energy window with respect to the Fermi level, is shown below $T_{r}$ in the $C_{4}$ magnetic phase at $23 \mathrm{~K}$, in Fig. 5(a). Two momentum cuts have been made along the $\Gamma \rightarrow$ M path; the first examines the temperature dependence of the anisotropic electron-like bands around an M point, Fig. 5(b), and the second details the behavior of the isotropic hole-like pockets around the $\Gamma$ point, shown in Fig. 5(c). This Fermi surface is qualitatively similar to what was observed in $\mathrm{Ba}_{1-x} \mathrm{~K}_{x} \mathrm{Fe}_{2} \mathrm{As}_{2}$ [70, 71]

At high temperature, the cut along the $\Gamma \rightarrow$ M direction at the $\mathrm{M}$ point there appears to be a hole-like band as well as a possible electron-like band at $135 \mathrm{~K}$, shown in the upper panel of Fig. 5(b). In the simple picture for the Fermi surface of $\mathrm{SrFe}_{2} \mathrm{As}_{2}$ (supplementary Fig. S4) this result can be reproduced by lowering the Fermi level $\epsilon_{F}$ by about $0.2 \mathrm{eV}$, which is consistent with the removal of electrons due to sodium substitution (hole doping). As the temperature is lowered below $T_{\mathrm{N}}$ and enters the magnetic $C_{2}$ phase, the hole-like band may split, while the electron-like band appears to shift below $\epsilon_{F}$. Below $T_{r}$ in the $C_{4}$ magnetic phase, a single hole-like band is recovered, while the electron-like band now appears to be split into two bands, with a separation of $\simeq 20 \mathrm{meV}$, which is 
comparable to the position of the low-energy peak (this behavior is explored further in supplementary Fig. S5).

The initial investigation into the temperature dependence of the energy bands around the $\Gamma$ point in Fig. 5 (c) revealed two large hole pockets at the Fermi level, but relatively little temperature dependence. This prompted a more detailed investigation of the hole-like bands along the $\Gamma \rightarrow$ M path, shown in Fig. 6 (further detail is provided in supplementary Figs. S6 and S7). Above $T_{\mathrm{N}}$ the bands are rather broad, but at least three bands may be resolved, all of which cross the Fermi level, resulting in several large hole-like Fermi surfaces, shown in the second-derivative curves in Fig. 6(a). Below $T_{\mathrm{N}}$ the bands sharpen considerably in the $C_{2}$ phase, and one of the bands is observed to shift to $\simeq 40 \mathrm{meV}$ below the Fermi level, shown in Fig. 6(b), leading to the removal of a hole-like Fermi surface; this is consistent with the Fermi surface reconstruction below $T_{\mathrm{N}}$ observed in the parent compounds [58, 72. This trend continues in the magnetic $C_{4}$ phase, with the band shifting to $\simeq 60 \mathrm{meV}$ below the Fermi level, Fig. 6(c).

\section{Discussion}

Both the electron and hole pockets appear to undergo significant changes in response to the Fermi surface reconstruction in the magnetic $C_{2}$ and $C_{4}$ phases that exhibit SDW and CSDW order, respectively. In the case of the hole pockets, the fact that one of the bands shifts below $\epsilon_{F}$ below $T_{\mathrm{N}}$ in the magnetic $C_{2}$ phase, shifting further below $T_{r}$ in the magnetic $C_{4}$ phase, signals the decrease in the size of the Fermi surface associated with the hole pockets. It is possible that this may be related to the dramatic decrease in the spectral weight of the broad Drude component as described by the plasma frequency in Fig. $4(\mathrm{a})$; from $\omega_{p, D 2}^{2} \propto n / m^{*}$ we infer a significant decrease in the carriers associated with the hole pockets at low temperature $(\simeq 90 \%$ reduction of the room temperature value).

The evolution of the electron-like bands is more complicated, as the bands at the $\mathrm{M}$ point have both electronand hole-like character. The initial splitting of the holelike band below $T_{\mathrm{N}}$ is consistent with the lifting of the degeneracy between the $d_{x z}$ and $d_{y z}$ orbitals; however, the fact that one of the hole-like bands lies completely below the Fermi level suggests no significant changes to the size of the Fermi surfaces. Below $T_{r}$ the orbital degeneracy is restored, but the presence of CSDW order leads to the formation of a supercell; the electron-like bands are split as a result of zone-folding, which may lead to an increase in the size of the Fermi surface. This is consistent with the slight increase in the plasma frequency of the narrow Drude component at low temperature, shown in Fig. 4(a). Furthermore, the splitting between the two electron-like bands of $\simeq 20 \mathrm{meV}$, is very close to the position of the low-energy peak. This suggests that, similar to the mid-infrared peak, the low-energy peak emerges in response to the Fermi surface reconstruction driven by the $C_{4}$ magnetic phase and the CSDW order at low temperature 43.

\section{SUMMARY}

The ARPES and complex optical properties of freshly-cleaved surfaces of the iron-based superconductor $\mathrm{Sr}_{0.67} \mathrm{Na}_{0.33} \mathrm{Fe}_{2} \mathrm{As}_{2}$ have been determined for light polarized in the iron-arsenic $(a-b)$ planes at a variety of temperatures for the room temperature tetragonal paramagnetic phase, the orthorhombic $C_{2}$ SDW magnetic phase, the tetragonal $C_{4}$ double-Q SDW (CSDW) phase, as well as below $T_{c}$ in the superconducting state. The freecarrier response is described by two Drude components, one broad and strong, the other narrow and weak. The strength of the narrow component shows little temperature dependence, increasing slightly in strength at low temperature, while narrowing dramatically. The broad Drude component decreases dramatically in strength and narrows below $T_{\mathrm{N}}$ at the same time a peak emerges in the mid-infrared; the decrease in the spectral weight associated with the free carriers is transferred into the emergent peak. Below $T_{r}$, this trend continues, with the emergence of a new low-energy peak at $\simeq 20 \mathrm{meV}$. The appearance of a new infrared-active mode in the $\mathrm{Fe}$-As planes below $T_{r}$ is attributed to zone-folding due to the formation of a supercell in response to the CSDW; this suggests that the low-energy peak originates from a further Fermi surface reconstruction in the $C_{4}$ phase. Below $T_{c}$ the lowfrequency conductivity decreases dramatically, signalling the formation of a superconducting energy gap. ARPES reveals large hole-like Fermi surfaces at the $\Gamma$ point, one of which is apparently removed below the structural and magnetic transitions, suggesting that they may be related to the behavior of the broad Drude component. The electron- and hole-like bands at the corners of the Brillouin zone shift and split below $T_{\mathrm{N}}$ and $T_{r}$, but the Fermi surfaces do not appear to undergo any significant change in size, suggesting they may be related to the narrow Drude component; the apparent splitting of the electronlike bands in the $C_{4}$ phase would appear to explain the emergence of the low-energy peak at $\simeq 20 \mathrm{meV}$ in the optical conductivity. While the $C_{2}$ and $C_{4}$ magnetic transitions, with resulting SDW and CSDW order, respectively, lead to a significant reconstruction of the Fermi surface that has profound implications for the transport originating from the electron- and hole-like pockets, they appear to have relatively little impact on the superconductivity in this material.

\section{ACKNOWLEDGMENTS}

Work at Chinese Academy of Science was supported by NSFC (Project Nos. 11774400, 11888101, and 11974412) and MOST (Project Nos. 2015CB921102, 2016YFA0300300, and 2017YFA0302903). Work at HP- 
STAR was supported by NSAF, Grant No. U1530402. Work at Brookhaven National Laboratory was supported by the Office of Science, U.S. Department of Energy under Contract No. DE-SC0012704.
[1] David C. Johnston, "The puzzle of high temperature superconductivity in layered iron pnictides and chalcogenides," Adv. Phys. 59, 803-1061 (2010).

[2] Johnpierre Paglione and Richard L. Greene, "Hightemperature superconductivity in iron-based materials," Nat. Phys. 6, 645-658 (2010)

[3] Paul C. Canfield and Sergey L. Bud'ko, "FeAs-Based Superconductivity: A Case Study of the Effects of Transition Metal Doping on $\mathrm{BaFe}_{2} \mathrm{As}_{2}$," Ann. Rev. Cond. Mat. Phys. 1, 27-50 (2010)

[4] Qimiao Si, Rong Yu, and Elihu Abrahams, "Hightemperature superconductivity in iron pnictides and chalcogenides," Nat. Rev. Mater. 1, 16017 (2016).

[5] M. P. M. Dean, M. G. Kim, A. Kreyssig, J. W. Kim, X. Liu, P. J. Ryan, A. Thaler, S. L. Bud'ko, W. Strassheim, P. C. Canfield, J. P. Hill, and A. I. Goldman, "Magnetically polarized Ir dopant atoms in superconducting $\mathrm{Ba}\left(\mathrm{Fe}_{1-x} \mathrm{Ir}_{x}\right)_{2} \mathrm{As}_{2}$," Phys. Rev. B 85, 140514(R) (2012)

[6] Pengcheng Dai, "Antiferromagnetic order and spin dynamics in iron-based superconductors," Rev. Mod. Phys. 87, 855-896 (2015).

[7] M. Moroni, P. Carretta, G. Allodi, R. De Renzi, M. N. Gastiasoro, B. M. Andersen, P. Materne, H.-H. Klauss, Y. Kobayashi, M. Sato, and S. Sanna, "Fast recovery of the stripe magnetic order by $\mathrm{Mn} / \mathrm{Fe}$ substitution in F-doped LaFeAsO superconductors," Phys. Rev. B 95, 180501(R) (2017)

[8] A. Kreyssig, J. M. Wilde, A. E. Böhmer, W. Tian, W. R. Meier, Bing Li, B. G. Ueland, Mingyu Xu, S. L. Bud'ko, P. C. Canfield, R. J. McQueeney, and A. I. Goldman, "Antiferromagnetic order in $\mathrm{CaK}\left(\mathrm{Fe}_{1-x} \mathrm{Ni}_{x}\right)_{4} \mathrm{As}_{4}$ and its interplay with superconductivity," Phys. Rev. B 97, 224521 (2018).

[9] William R. Meier, Qing-Ping Ding, Andreas Kreyssig, Sergey L. Bud'ko, Aashish Sapkota, Karunakar Kothapalli, Vladislav Borisov, Roser Valentí, Cristian D. Batista, Peter P. Orth, Rafael M. Fernandes, Alan I. Goldman, Yuji Furukawa, Anna E. Böhmer, and Paul C. Canfield, "Hedgehog spin-vortex crystal stabilized in a hole-doped iron-based superconductor," npj Quantum Materials 3, 5 (2018).

[10] Marianne Rotter, Marcus Tegel, and Dirk Johrendt, "Superconductivity at $38 \mathrm{~K}$ in the Iron Arsenide $\left(\mathrm{Ba}_{1-x} \mathrm{~K}_{x}\right) \mathrm{Fe}_{2} \mathrm{As}_{2}, "$ Phys. Rev. Lett. 101, 107006 (2008).

[11] Athena S. Sefat, Rongying Jin, Michael A. McGuire, Brian C. Sales, David J. Singh, and David Mandrus, "Superconductivity at $22 \mathrm{~K}$ in Co-Doped $\mathrm{BaFe}_{2} \mathrm{As}_{2}$ Crystals," Phys. Rev. Lett. 101, 117004 (2008).

[12] N. Ni, M. E. Tillman, J.-Q. Yan, A. Kracher, S. T. Hannahs, S. L. Bud'ko, and P. C. Canfield, "Effects of Co substitution on thermodynamic and transport properties and anisotropic $H_{c 2}$ in $\mathrm{Ba}\left(\mathrm{Fe}_{1-x} \mathrm{Co}_{x}\right)_{2} \mathrm{As}_{2}$ single crystals," Phys. Rev. B 78, 214515 (2008).

[13] Kalyan Sasmal, Bing Lv, Bernd Lorenz, Arnold M. Guloy, Feng Chen, Yu-Yi Xue, and Ching-Wu Chu, "Superconducting Fe-Based Compounds $\left(A_{1-x} \mathrm{Sr}_{x}\right) \mathrm{Fe}_{2} \mathrm{As}_{2}$ with
$A=\mathrm{K}$ and Cs with Transition Temperatures up to $37 \mathrm{~K}$," Phys. Rev. Lett. 101, 107007 (2008)

[14] Gen-Fu Chen, Zheng Li, Gang Li, Wan-Zheng Hu, Jing Dong, Xiao-Dong Zhang Jun Zhou, Ping Zheng, Nan-Lin Wang, and Jian-Lin Luo, "Superconductivity in HoleDoped $\left(\mathrm{Sr}_{1-x} \mathrm{~K}_{x}\right) \mathrm{Fe}_{2} \mathrm{As}_{2}$," Chin. Phys. Lett. 25, 3403 (2008)

[15] Jiun-Haw Chu, James G. Analytis, Chris Kucharczyk, and Ian R. Fisher, "Determination of the phase diagram of the electron-doped superconductor $\mathrm{Ba}\left(\mathrm{Fe}_{1-x} \mathrm{Co}_{x}\right)_{2} \mathrm{As}_{2}$," Phys. Rev. B 79, 014506 (2009).

[16] T. Goko, A. A. Aczel, E. Baggio-Saitovitch, S. L. Bud'ko, P. C. Canfield, J. P. Carlo, G. F. Chen, Pengcheng Dai, A. C. Hamann, W. Z. Hu, H. Kageyama, G. M. Luke, J. L. Luo, B. Nachumi, N. Ni, D. Reznik, D. R. SanchezCandela, A. T. Savici, K. J. Sikes, N. L. Wang, C. R. Wiebe, T. J. Williams, T. Yamamoto, W. Yu, and Y. J. Uemura, "Superconducting state coexisting with a phase-separated static magnetic order in $(\mathrm{Ba}, \mathrm{K}) \mathrm{Fe}_{2} \mathrm{As}_{2}$, $(\mathrm{Sr}, \mathrm{Na}) \mathrm{Fe}_{2} \mathrm{As}_{2}$, and $\mathrm{CaFe}_{2} \mathrm{As}_{2}$," Phys. Rev. B 80, 024508 (2009)

[17] S. R. Saha, N. P. Butch, K. Kirshenbaum, and Johnpierre Paglione, "Evolution of bulk superconductivity in $\mathrm{SrFe}_{2} \mathrm{As}_{2}$ with Ni substitution," Phys. Rev. B 79, 224519 (2009)

[18] Shuai Jiang, Hui Xing, Guofang Xuan, Cao Wang, Zhi Ren, Chunmu Feng, Jianhui Dai, Zhu'an Xu, and Guanghan Cao, "Superconductivity up to $30 \mathrm{~K}$ in the vicinity of the quantum critical point in $\mathrm{BaFe}_{2}\left(\mathrm{As}_{1-x} \mathrm{P}_{x}\right)_{2}$," J. Phys.: Condens. Matter 21, 382203 (2009)

[19] H. L. Shi, H. X. Yang, H. F. Tian, J. B. Lu, Z. W. Wang, Y. B. Qin, Y. J. Song, and J. Q. Li, "Structural properties and superconductivity of $\mathrm{SrFe}_{2} \mathrm{As}_{2-x} \mathrm{P}_{x}$ and $(0.0 \leq x \leq 1.0)$ and $\mathrm{CaFe}_{2} \mathrm{As}_{2-y} \mathrm{P}_{y}(0.0 \leq y \leq 0.3)$," J. Phys.: Condens. Matter 22, 125702 (2010).

[20] Raquel Cortes-Gil and Simon J. Clarke, "Structure, Magnetism, and Superconductivity of the Layered Iron Arsenides $\mathrm{Sr}_{1-x} \mathrm{Na}_{x} \mathrm{Fe}_{2} \mathrm{As}_{2}$," Chem. Mater. 23, 1009-1016 (2011)

[21] Fumihiro Ishikawa, Naoya Eguchi, Michihiro Kodama, Koji Fujimaki, Mari Einaga, Ayako Ohmura, Atsuko Nakayama, Akihiro Mitsuda, and Yuh Yamada, "Zeroresistance superconducting phase in $\mathrm{BaFe}_{2} \mathrm{As}_{2}$ under high pressure," Phys. Rev. B 79, 172506 (2009).

[22] Patricia L. Alireza, Y. T. Chris Ko, Jack Gillett, Chiara M. Petrone, Jacqui M. Cole, Suchitra E. Sebastian, and Gilbert G. Lonzarich, "Superconductivity up to $29 \mathrm{~K}$ in $\mathrm{SrFe}_{2} \mathrm{As}_{2}$ and $\mathrm{BaFe}_{2} \mathrm{As}_{2}$ at high pressures," J. Phys: Cond. Matter 21, 012208 (2009)

[23] E. Colombier, S. L. Bud'ko, N. Ni, and P. C. Canfield, "Complete pressure-dependent phase diagrams for $\mathrm{SrFe}_{2} \mathrm{As}_{2}$ and $\mathrm{BaFe}_{2} \mathrm{As}_{2}$," Phys. Rev. B 79, 224518 (2009).

[24] K. Kitagawa, N. Katayama, H. Gotou, T. Yagi, K. Ohgushi, T. Matsumoto, Y. Uwatoko, and M. Takigawa, "Spontaneous Formation of a Superconducting and Anti- 
ferromagnetic Hybrid State in $\mathrm{SrFe}_{2} \mathrm{As}_{2}$ under High Pressure," Phys. Rev. Lett. 103, 257002 (2009).

[25] Marcus Tegel, Marianne Rotter, Veronika Wei $\beta$, Falko M Schappacher, Rainer Pöttgen, and Dirk Johrendt, "Structural and magnetic phase transitions in the ternary iron arsenides $\mathrm{SrFe}_{2} \mathrm{As}_{2}$ and $\mathrm{EuFe}_{2} \mathrm{As}_{2}$," J. Phys.: Condens. Matter 20, 452201 (2008)

[26] J.-Q. Yan, A. Kreyssig, S. Nandi, N. Ni, S. L. Bud'ko, A. Kracher, R. J. McQueeney, R. W. McCallum, T. A. Lograsso, A. I. Goldman, and P. C. Canfield, "Structural transition and anisotropic properties of single-crystalline $\mathrm{SrFe}_{2} \mathrm{As}_{2}$," Phys. Rev. B 78, 024516 (2008).

[27] Jun Zhao, W. Ratcliff, J. W. Lynn, G. F. Chen, J. L. Luo, N. L. Wang, Jiangping $\mathrm{Hu}$, and Pengcheng Dai, "Spin and lattice structures of single-crystalline $\mathrm{SrFe}_{2} \mathrm{As}_{2}$," Phys. Rev. B 78, 140504(R) (2008)

[28] W. Z. Hu, J. Dong, G. Li, Z. Li, P. Zheng, G. F. Chen, J. L. Luo, and N. L. Wang, "Origin of the Spin Density Wave Instability in $A \mathrm{Fe}_{2} \mathrm{As}_{2}(A=\mathrm{Ba}, \mathrm{Sr})$ as Revealed by Optical Spectroscopy," Phys. Rev. Lett. 101, 257005 (2008)

[29] J. N. Hancock, S. I. Mirzaei, J. Gillett, S. E. Sebastian, J. Teyssier, R. Viennois, E. Giannini, and D. van der Marel, "Strong coupling to magnetic fluctuations in the charge dynamics of iron-based superconductors," Phys. Rev. B 82, 014523 (2010).

[30] E. C. Blomberg, M. A. Tanatar, A. Kreyssig, N. Ni, A. Thaler, Rongwei Hu, S. L. Bud'ko, P. C. Canfield, A. I. Goldman, and R. Prozorov, "In-plane anisotropy of electrical resistivity in strain-detwinned $\mathrm{SrFe}_{2} \mathrm{As}_{2}$," Phys. Rev. B 83, 134505 (2011).

[31] M. A. Tanatar, A. Kreyssig, S. Nandi, N. Ni, S. L. Bud'ko, P. C. Canfield, A. I. Goldman, and R. Prozorov, "Direct imaging of the structural domains in the iron pnictides $A \mathrm{Fe}_{2} \mathrm{As}_{2}(A=\mathrm{Ca}, \mathrm{Sr}, \mathrm{Ba})$," Phys. Rev. B 79, 180508(R) (2009)

[32] I. R. Fisher, L. Degiorgi, and Z. X. Shen, "In-plane electronic anisotropy of underdoped ' 122 ' Fe-arsenide superconductors revealed by measurements of detwinned single crystals," Rep. Prog. Phys. 74, 124506 (2011)

[33] A. I. Goldman, D. N. Argyriou, B. Ouladdiaf, T. Chatterji, A. Kreyssig, S. Nandi, N. Ni, S. L. Bud'ko, P. C. Canfield, and R. J. McQueeney, "Lattice and magnetic instabilities in $\mathrm{CaFe}_{2} \mathrm{As}_{2}$ : A single-crystal neutron diffraction study," Phys. Rev. B 78, 100506(R) (2008)

[34] M. Kofu, Y. Qiu, Wei Bao, S.-H. Lee, S. Chang, T. Wu, G. Wu, and X. H. Chen, "Neutron scattering investigation of the magnetic order in single crystalline $\mathrm{BaFe}_{2} \mathrm{As}_{2}$," New J. Phys. 11, 055001 (2009).

[35] M. G. Kim, A. Kreyssig, A. Thaler, D. K. Pratt, W. Tian, J. L. Zarestky, M. A. Green, S. L. Bud'ko, P. C. Canfield, R. J. McQueeney, and A. I. Goldman, "Antiferromagnetic ordering in the absence of structural distortion in $\mathrm{Ba}\left(\mathrm{Fe}_{1-x} \mathrm{Mn}_{x}\right)_{2} \mathrm{As}_{2}$," Phys. Rev. B 82, 220503(R) (2010)

[36] E. Hassinger, G. Gredat, F. Valade, S. René de Cotret, A. Juneau-Fecteau, J.-Ph. Reid, H. Kim, M. A. Tanatar, R. Prozorov, B. Shen, H.-H. Wen, N. DoironLeyraud, and Louis Taillefer, "Pressure-induced Fermisurface reconstruction in the iron-arsenide superconductor $\mathrm{Ba}_{1-x} \mathrm{~K}_{x} \mathrm{Fe}_{2} \mathrm{As}_{2}$ : Evidence of a phase transition inside the antiferromagnetic phase," Phys. Rev. B 86, 140502(R) (2012)
[37] A. E. Böhmer, F. Hardy, L. Wang, T. Wolf, P. Schweiss, and C. Meingast, "Superconductivityinduced re-entrance of the orthorhombic distortion in $\mathrm{Ba}_{1-x} \mathrm{~K}_{x} \mathrm{Fe}_{2} \mathrm{As}_{2}$," Nat. Commun. 6, 7911 (2015).

[38] L. Wang, F. Hardy, A. E. Böhmer, T. Wolf, P. Schweiss, and C. Meingast, "Complex phase diagram of $\mathrm{Ba}_{1-x} \mathrm{Na}_{x} \mathrm{Fe}_{2} \mathrm{As}_{2}$ : A multitude of phases striving for the electronic entropy," Phys. Rev. B 93, 014514 (2016)

[39] K. M. Taddei, J. M. Allred, D. E. Bugaris, S. Lapidus, M. J. Krogstad, R. Stadel, H. Claus, D. Y. Chung, M. G. Kanatzidis, S. Rosenkranz, R. Osborn, and O. Chmaissem, "Detailed magnetic and structural analysis mapping a robust magnetic $C_{4}$ dome in $\mathrm{Sr}_{1-x} \mathrm{Na}_{x} \mathrm{Fe}_{2} \mathrm{As}_{2}$," Phys. Rev. B 93, 134510 (2016).

[40] Liran Wang, Mingquan He, Daniel D. Scherer, Frédéric Hardy, Peter Schweiss, Thomas Wolf, Michael Merz, Brian M. Andersen, and Christoph Meingast, "Competing Electronic Phases near the Onset of Superconductivity in Hole-doped $\mathrm{SrFe}_{2} \mathrm{As}_{2}$," J. Phys. Soc. Jpn. 88, 104710 (2019)

[41] E. Hassinger, G. Gredat, F. Valade, S. René de Cotret, O. Cyr-Choinière, A. Juneau-Fecteau, J.-Ph. Reid, H. Kim, M. A. Tanatar, R. Prozorov, B. Shen, H.-H. Wen, N. Doiron-Leyraud, and Louis Taillefer, "Expansion of the tetragonal magnetic phase with pressure in the iron arsenide superconductor $\mathrm{Ba}_{1-x} \mathrm{~K}_{x} \mathrm{Fe}_{2} \mathrm{As}_{2}$," Phys. Rev. B 93, 144401 (2016).

[42] K. M. Taddei, J. M. Allred, D. E. Bugaris, S. H. Lapidus, M. J. Krogstad, H. Claus, D. Y. Chung, M. G. Kanatzidis, R. Osborn, S. Rosenkranz, and O. Chmaissem, "Observation of the magnetic $C_{4}$ phase in $\mathrm{Ca}_{1-x} \mathrm{Na}_{x} \mathrm{Fe}_{2} \mathrm{As}_{2}$ and its universality in the hole-doped 122 superconductors," Phys. Rev. B 95, 064508 (2017).

[43] M. Yi, A. Frano, D. H. Lu, Y. He, Meng Wang, B. A. Frandsen, A. F. Kemper, R. Yu, Q. Si, L. Wang, M. He, F. Hardy, P. Schweiss, P. Adelmann, T. Wolf, M. Hashimoto, S.-K. Mo, Z. Hussain, M. Le Tacon, A. E. Böhmer, D.-H. Lee, Z.-X. Shen, C. Meingast, and R. J. Birgeneau, "Spectral Evidence for Emergent Order in $\mathrm{Ba}_{1-x} \mathrm{Na}_{x} \mathrm{Fe}_{2} \mathrm{As}_{2}$," Phys. Rev. Lett. 121, 127001 (2018).

[44] S. Avci, O. Chmaissem, J. M. Allred, S. Rosenkranz, I. Eremin, A. V. Chubukov, D. E. Bugaris, D. Y. Chung, M. G. Kanatzidis, J.-P Castellan, J. A. Schlueter, H. Claus, D. D. Khalyavin, P. Manuel, A. DaoudAladine, and R. Osborn, "Magnetically driven suppression fo nematic order in an iron-based superconductor," Nat. Commun. 5, 3845 (2014)

[45] J. M. Allred, K. M. Taddei, D. E. Bugaris, M. J. Krogstad, S. H. Lapidus, D. Y. Chung, H. Claus, M. G. Kanatzidis, D. E. Brown, J. Kang, R. M. Fernandes, I. Eremin, S. Rosenkranz, O. Chmaissem, and R. Osborn, "Double-Q spin-density wave in iron arsenide superconductors," Nat. Phys. 12, 493 (2016)

[46] F. Waßer, A. Schneidewind, Y. Sidis, S. Wurmehl, S. Aswartham, B. Büchner, and M. Braden, "Spin reorientation in $\mathrm{Ba}_{0.65} \mathrm{Na}_{0.35} \mathrm{Fe}_{2} \mathrm{As}_{2}$ studied by single-crystal neutron diffraction," Phys. Rev. B 91, 060505(R) (2015).

[47] B. P. P. Mallett, Yu. G. Pashkevich, A. Gusev, Th. Wolf, and C. Bernhard, "Muon spin rotation study of the magnetic structure in the tetragonal antiferromagnetic state of weakly underdoped $\mathrm{Ba}_{1-x} \mathrm{~K}_{x} \mathrm{Fe}_{2} \mathrm{As}_{2}$," $\mathrm{EPL}$ (Europhysics Letters) 111, 57001 (2015)

[48] Mareike Hoyer, Rafael M. Fernandes, Alex Levchenko, and Jörg Schmalian, "Disorder-promoted $C_{4}$-symmetric 
magnetic order in iron-based superconductors," Phys. Rev. B 93, 144414 (2016).

[49] Jianqing Guo, Li Yue, Kazuki Iida, Kazuya Kamazawa, Lei Chen, Tingting Han, Yan Zhang, and Yuan $\mathrm{Li}$, "Preferred magnetic excitations in the iron-based $\mathrm{Sr}_{1-x} \mathrm{Na}_{x} \mathrm{Fe}_{2} \mathrm{As}_{2}$ superconductor," Phys. Rev. Lett. 122, 017001 (2019)

[50] Christopher C. Homes, M. Reedyk, D. A. Crandles, and T. Timusk, "Technique for measuring the reflectance of irregular, submillimeter-sized samples," Appl. Opt. 32, 2976-2983 (1993)

[51] See Supplemental Material at [URL will be inserted by publisher] for details of the experimental reflectivity and Kramers-Kronig analysis, which includes Refs. 73.77.

[52] Y. M. Dai, Ana Akrap, S. L. Bud'ko, P. C. Canfield, and C. C. Homes, "Optical properties of $A \mathrm{Fe}_{2} \mathrm{As}_{2}(A=\mathrm{Ca}$, Sr, and Ba) single crystals," Phys. Rev. B 94, 195142 (2016)

[53] C. C. Homes, Y. M. Dai, Ana Akrap, S. L. Bud'ko, and P. C. Canfield, "Vibrational anomalies in $A \mathrm{Fe}_{2} \mathrm{As}_{2}(A=$ $\mathrm{Ca}, \mathrm{Sr}$, and Ba) single crystals," Phys. Rev. B 98, 035103 (2018)

[54] B. P. P. Mallett, P. Marsik, M. Yazdi-Rizi, Th. Wolf, A. E. Böhmer, F. Hardy, C. Meingast, D. Munzar, and C. Bernhard, "Infrared Study of the Spin Reorientation Transition and Its Reversal in the Superconducting State in Underdoped $\mathrm{Ba}_{1-x} \mathrm{~K}_{x} \mathrm{Fe}_{2} \mathrm{As}_{2}$," Phys. Rev. Lett. 115, $027003(2015)$

[55] D. J. Singh, "Electronic structure and doping in $\mathrm{BaFe}_{2} \mathrm{As}_{2}$ and LiFeAs: Density functional calculations," Phys. Rev. B 78, 094511 (2008)

[56] J. Fink, S. Thirupathaiah, R. Ovsyannikov, H. A. Dürr, R. Follath, Y. Huang, S. de Jong, M. S. Golden, YuZhong Zhang, H. O. Jeschke, R. Valentí, C. Felser, S. Dastjani Farahani, M. Rotter, and D. Johrendt, "Electronic structure studies of $\mathrm{BaFe}_{2} \mathrm{As}_{2}$ by angle-resolved photoemission spectroscopy," Phys. Rev. B 79, 155118 (2009)

[57] D. Wu, N. Barišić, P. Kallina, A. Faridian, B. Gorshunov, N. Drichko, L. J. Li, X. Lin, G. H. Cao, Z. A. Xu, N. L. Wang, and M. Dressel, "Optical investigations of the normal and superconducting states reveal two electronic subsystems in iron pnictides," Phys. Rev. B 81, 100512(R) (2010)

[58] Z. P. Yin, K. Haule, and G. Kotliar, "Magnetism and charge dynamics in iron pnictides," Nat. Phys. 7, 294297 (2011)

[59] Richard A. Ferrell and Rolfe E. Glover, "Conductivity of Superconducting Films: A Sum Rule," Phys. Rev. 109, 1398-1399 (1958)

[60] M. Tinkham and R. A. Ferrell, "Determination of the Superconducting Skin Depth from the Energy Gap and Sum Rule," Phys. Rev. Lett. 2, 331-333 (1959).

[61] C. C. Homes, Y. M. Dai, J. S. Wen, Z. J. Xu, and G. D. $\mathrm{Gu}$, "FeTe ${ }_{0.55} \mathrm{Se}_{0.45}$ : A multiband superconductor in the clean and dirty limit," Phys. Rev. B 91, 144503 (2015).

[62] C. C. Homes, S. V. Dordevic, M. Strongin, D. A. Bonn, Ruixing Liang, W. N. Hardy, Seiki Komiya, Yoichi Ando, G. Yu, N. Kaneko, X. Zhao, M. Greven, D. N. Basov, and T. Timusk, "Universal scaling relation in hightemperature superconductors," Nature (London) 430, 539 (2004)

[63] C. C. Homes, S. V. Dordevic, D. A. Bonn, Ruixing Liang, W. N. Hardy, and T. Timusk, "Coherence, incoherence, and scaling along the $c$ axis of $\mathrm{YBa}_{2} \mathrm{Cu}_{3} \mathrm{O}_{6+x}$," Phys. Rev. B 71, 184515 (2005).

[64] C. C. Homes, S. V. Dordevic, T. Valla, and M. Strongin, "Scaling of the superfluid density in high-temperature superconductors," Phys. Rev. B 72, 134517 (2005).

[65] J. J. Tu, J. Li, W. Liu, A. Punnoose, Y. Gong, Y. H. Ren, L. J. Li, G. H. Cao, Z. A. Xu, and C. C. Homes, "Optical properties of the iron arsenic superconductor $\mathrm{BaFe}_{1.85} \mathrm{Co}_{0.15} \mathrm{As}_{2}$," Phys. Rev. B 82, 174509 (2010)

[66] Run Yang, Yaomin Dai, Jia Yu, Qiangtao Sui, Yongqing Cai, Zhian Ren, Jungseek Hwang, Hong Xiao, Xingjiang Zhou, Xianggang Qiu, and Christopher C. Homes, "Unravelling the mechanism of the semiconductinglike behavior and its relation to superconductivity in $\left(\mathrm{CaFe}_{1-x} \mathrm{Pt}_{x} \mathrm{As}\right)_{10} \mathrm{Pt}_{3} \mathrm{Ass}_{8}, "$ Phys. Rev. B 99, 144520 (2019)

[67] N. V. Smith, "Classical generalization of the Drude formula for the optical conductivity," Phys. Rev. B 64, 155106 (2001)

[68] Replacing the broad Drude term with the expression in Eq. (2) and fitting to the real and imaginary parts of the optical conductivity using a non-linear least-squares technique yields $\omega_{p} \simeq 5350 \mathrm{~cm}^{-1}, 1 / \tau \simeq 146 \mathrm{~cm}^{-1}$, and $c=-0.7$. The plasma frequency is larger because it now describes both the localized as well as free carriers; $\omega_{p}^{2} \simeq$ $\omega_{p, D 2}^{2}+\Omega_{0}^{2}$.

[69] Y. M. Dai, B. Xu, B. Shen, H. H. Wen, J. P. Hu, X. G. Qiu, and R. P. S. M. Lobo, "Pseudogap in underdoped $\mathrm{Ba}_{1-x} \mathrm{~K}_{x} \mathrm{Fe}_{2} \mathrm{As}_{2}$ as seen via optical conductivity," Phys. Rev. B 86, 100501(R) (2012).

[70] V. B. Zabolotnyy, D. S. Inosov, D. V. Evtushinsky, A. Koitzsch, A. A. Kordyuk, G. L. Sun, J. T. Park, D. Haug, V. Hinkov, A. V. Boris, C. T. Lin, M. Knupfer, A. N. Yaresko, B. Büchner, A. Varykhalov, R. Follath, and S. V. Borisenko, " $(\pi, \pi)$ electronic order in iron arsenide superconductors," Nature 457, 569-572 (2009).

[71] Gerald Derondeau, Federico Bisti, Masaki Kobayashi, Jürgen Braun, Hubert Ebert, Victor A. Rogalev, Ming Shi, Thorsten Schmitt, Junzhang Ma, Hong Ding, Vladimir N. Strocov, and Ján Minár, "Fermi surface and effective masses in photoemission response of the $\left(\mathrm{Ba}_{1-x} \mathrm{~K}_{x}\right) \mathrm{Fe}_{2} \mathrm{As}_{2}$ superconductor," Sci. Rep. 7, 8787 (2017)

[72] M. Yi, D. H. Lu, J. G. Analytis, J.-H. Chu, S.-K. Mo, R.-H. He, M. Hashimoto, R. G. Moore, I. I. Mazin, D. J. Singh, Z. Hussain, I. R. Fisher, and Z.-X. Shen, "Unconventional electronic reconstruction in undoped (Ba, $\mathrm{Sr}) \mathrm{Fe}_{2} \mathrm{As}_{2}$ across the spin density wave transition," Phys. Rev. B 80, 174510 (2009).

[73] F. Wooten, Optical Properties of Solids (Academic Press, New York, 1972) pp. 244-250.

[74] M. Dressel and G. Grüner, Electrodynamics of Solids (Cambridge University Press, Cambridge, 2001).

[75] D. J. Singh, Planewaves, Pseudopotentials and the $L A P W$ method (Kluwer Adademic, Boston, 1994).

[76] David Singh, "Ground-state properties of lanthanum: Treatment of extended-core states," Phys. Rev. B 43, 6388-6392 (1991)

[77] P. Blaha, K. Schwarz, G. K. H. Madsen, D. Kvasnicka and J. Luitz, WIEN2k, An augmented plane wave plus local orbitals program for calculating crystal properties (Techn. Universität Wien, Austria, 2001). 


\title{
Supplementary material for: Optical and photoemission investigation of structural and magnetic transitions in the iron-based superconductor $\mathrm{Sr}_{0.67} \mathrm{Na}_{0.33} \mathrm{Fe}_{2} \mathrm{As}_{2}$
}

\author{
R. Yang,,${ }^{1,2,3, *}$ J. W. Huang,,${ }^{2,3}$ N. Zaki ${ }^{1}$ I. Pletikosić,${ }^{1,4}$ Y. M. Dai, ${ }^{5}$ H. Xiao, ${ }^{6}$ \\ T. Valla, ${ }^{1}$ P. D. Johnson, ${ }^{1}$ X. J. Zhou, ${ }^{2,3,7,8}$ X. G. Qiu, ${ }^{2,3,7, \dagger}$ and C. C. Homes ${ }^{1, \ddagger}$ \\ ${ }^{1}$ Condensed Matter Physics and Materials Science Division, \\ Brookhaven National Laboratory, Upton, New York 11973, USA \\ ${ }^{2}$ Beijing National Laboratory for Condensed Matter Physics, \\ Institute of Physics, Chinese Academy of Sciences, Beijing 100190, China \\ ${ }^{3}$ School of Physical Sciences, University of Chinese Academy of Sciences, Beijing 100049, China \\ ${ }^{4}$ Department of Physics, Princeton University, Princeton, NJ 08544, USA \\ ${ }^{5}$ Center for Superconducting Physics and Materials, \\ National Laboratory of Solid State Microstructures and Department of Physics, Nanjing University, Nanjing 210093, China \\ ${ }^{6}$ Center for High Pressure Science and Technology Advanced Research, Beijing 100094, China \\ ${ }^{7}$ Songshan Lake Materials Laboratory, Dongguan 523808, China \\ ${ }^{8}$ Beijing Academy of Quantum Information Science, Beijing 100193, China
}

\section{EXPERIMENTAL DETAILS}

High-quality single crystals of $\mathrm{Sr}_{0.67} \mathrm{Na}_{0.33} \mathrm{Fe}_{2} \mathrm{As}_{2}$ with good cleavage planes (001) were synthesized using selfflux method. The resistivity measurements were performed using a Quantum Design Physical Property Measurement System (PPMS). The reflectivity from a freshly-cleaved surface has been measured at a near-normal angle of incidence using Fourier transform infrared spectrometers (Bruker Vertex 80v and IFS 113v) for light polarized in the $a-b$ plane using an in situ evaporation technique [1]. Data from $\sim 40$ to $42000 \mathrm{~cm}^{-1}$ were collected at different temperatures from $\sim 5$ to $300 \mathrm{~K}$ using an ARS Helitran open-flow cryostat. The optical conductivity has been determined from a Kramers-Kronig analysis of the reflectivity $R(\omega)$. Because the measurement is performed over a finite energy range, extrapolations are necessary for $\omega \rightarrow 0, \infty[2]$. Below the lowest measured frequency, for $T>T_{c}$ the Hagen-Rubens relation $[R(\omega)=1-A \sqrt{\omega}]$ for a metal is used, while for $T<T_{c}$, a superconducting response $\left[R(\omega)=1-\omega^{4}\right]$ is

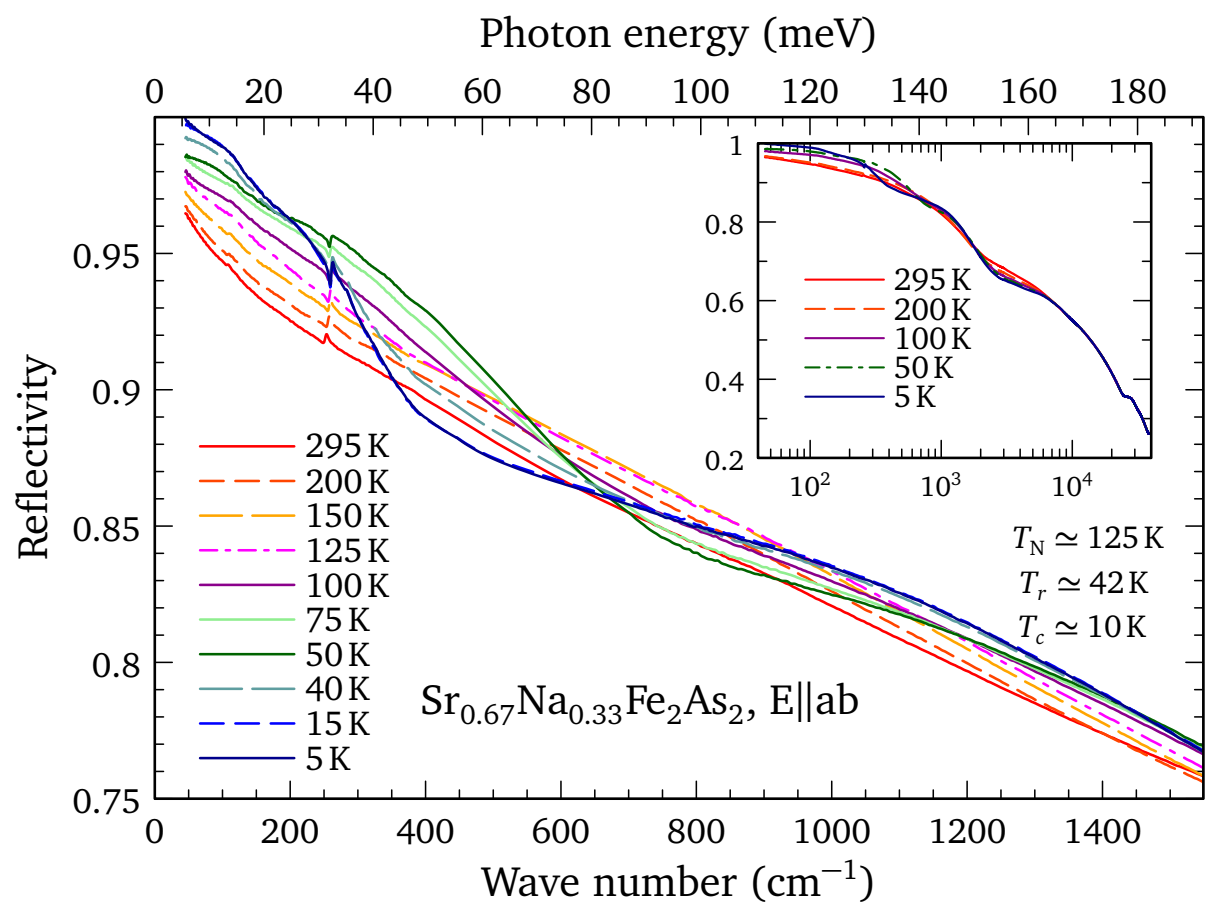

Figure S1. Temperature-dependent reflectivity of $\mathrm{Sr}_{0.67} \mathrm{Na}_{0.33} \mathrm{Fe}_{2} \mathrm{As}_{2}$ in the infrared region for light polarized in the Fe-As planes. Inset: the reflectivity in the high-frequency range at high and low temperatures. 


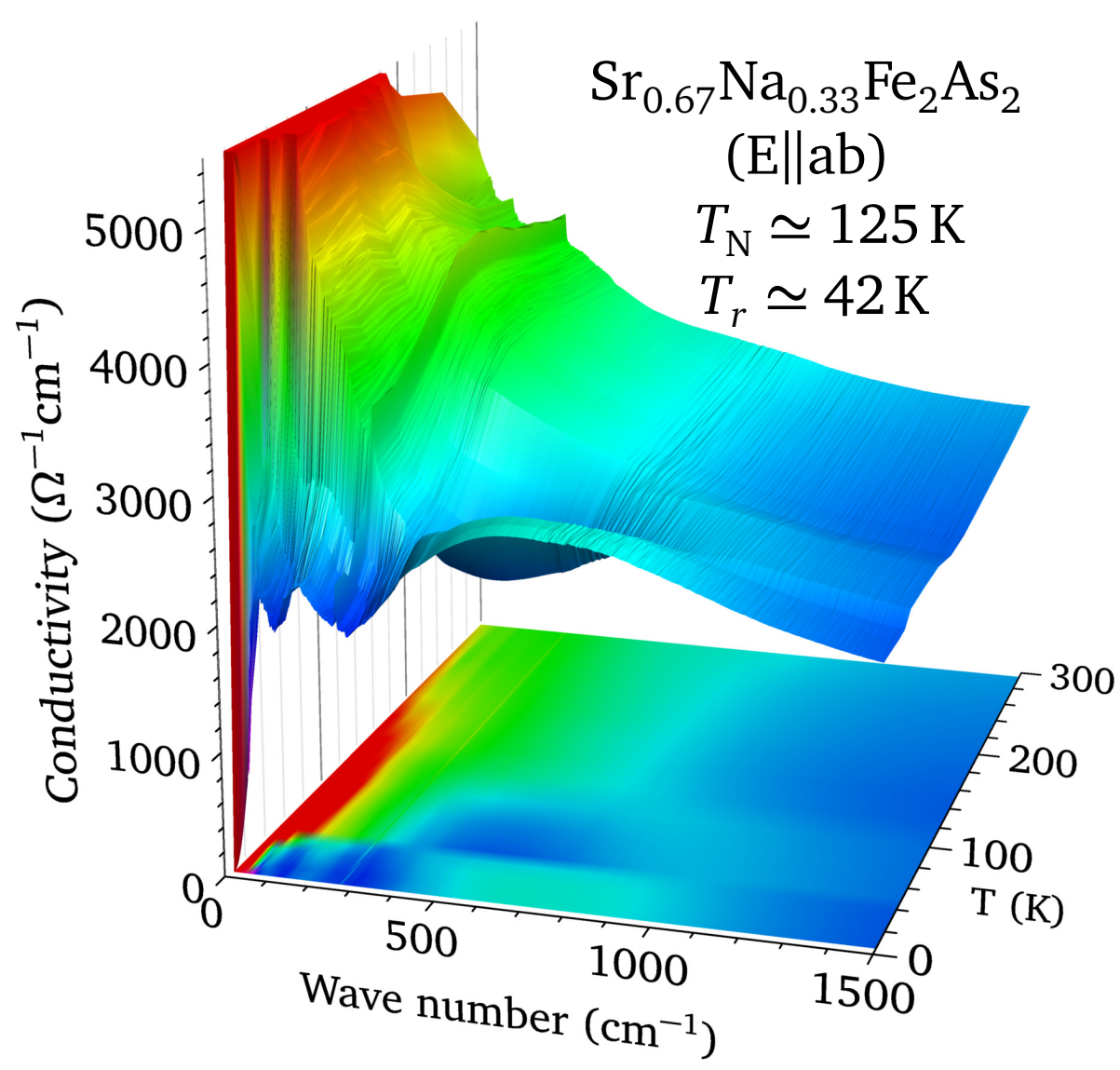

Figure S2. The real part of optical conductivity of $\mathrm{Sr}_{0.67} \mathrm{Na}_{0.33} \mathrm{Fe}_{2} \mathrm{As}_{2}$ in the infrared region for light polarized in the $a b$-planes shown as a surface plot, as well as a projection onto the temperature versus wave number plane.

employed. Above above the highest-measured frequency, $R(\omega)$ taken to be a constant up to $9 \mathrm{eV}$, above which a free-electron response $\left(\propto \omega^{-4}\right)$ is used [3].

Figure S1 shows the temperature dependence of the reflectivity $R(T, \omega)$ of $\mathrm{Sr}_{0.67} \mathrm{Na}_{0.33} \mathrm{Fe}_{2} \mathrm{As}_{2}$ over much of the infrared region; the inset shows the reflectivity over a much wider range at selected temperatures. Between room temperature and $T_{N}$ the reflectivty increases with decreasing temperature over much of the infrared region. For $T_{r}<T<T_{N}$, while the low-frequency reflectivity continues to increase, there is a suppression of the reflectance above about $600 \mathrm{~cm}^{-1}$. Finally, for $T<T_{r}$, while the trend of the increasing low-frequency reflectivity continues, the suppression of the reflectivity shifts now begins at about $300 \mathrm{~cm}^{-1}$. Below $T<T_{c}$ there is a slight upturn in the low-frequency reflectivity, corresponding to the superconducting transition observed in the resistivity (Fig. 1).

The real part of the optical conductivity determined from the Kramers-Kronig analysis of the reflectance is shown in the infrared region in Fig. S2 as a surface plot, as well as a projection onto the temperature versus wave number plane. The conductivity shows a broad Drude-like response in the high-temperature tetragonal paramagnetic metal phase that is actually described by two Drude components. Below $T_{N}$, the Drude-like response narrows and at the same time a peak emerges in the mid-infrared region at about $120 \mathrm{meV}$. As the temperature is reduced below $T_{r}$, the narrow Drude response decreases to the point where only the broad Drude response may be observed; at the same time, the mid-infrared peak shifts down to about $80 \mathrm{meV}$, and a new weak feature emerges at $\simeq 20 \mathrm{meV}$. Below $T_{c}$, there is a dramatic suppression of the low-frequency conductivity, signalling the formation of a superconducting energy gap; the missing spectral weight is transferred into the superconducting condensate. From Fig. S2, as well as the inset of Fig. 2 in the manuscript, the characteristic energy for the superconducting energy gap is estimated be $2 \Delta \simeq 50 \mathrm{~cm}^{-1}$. 


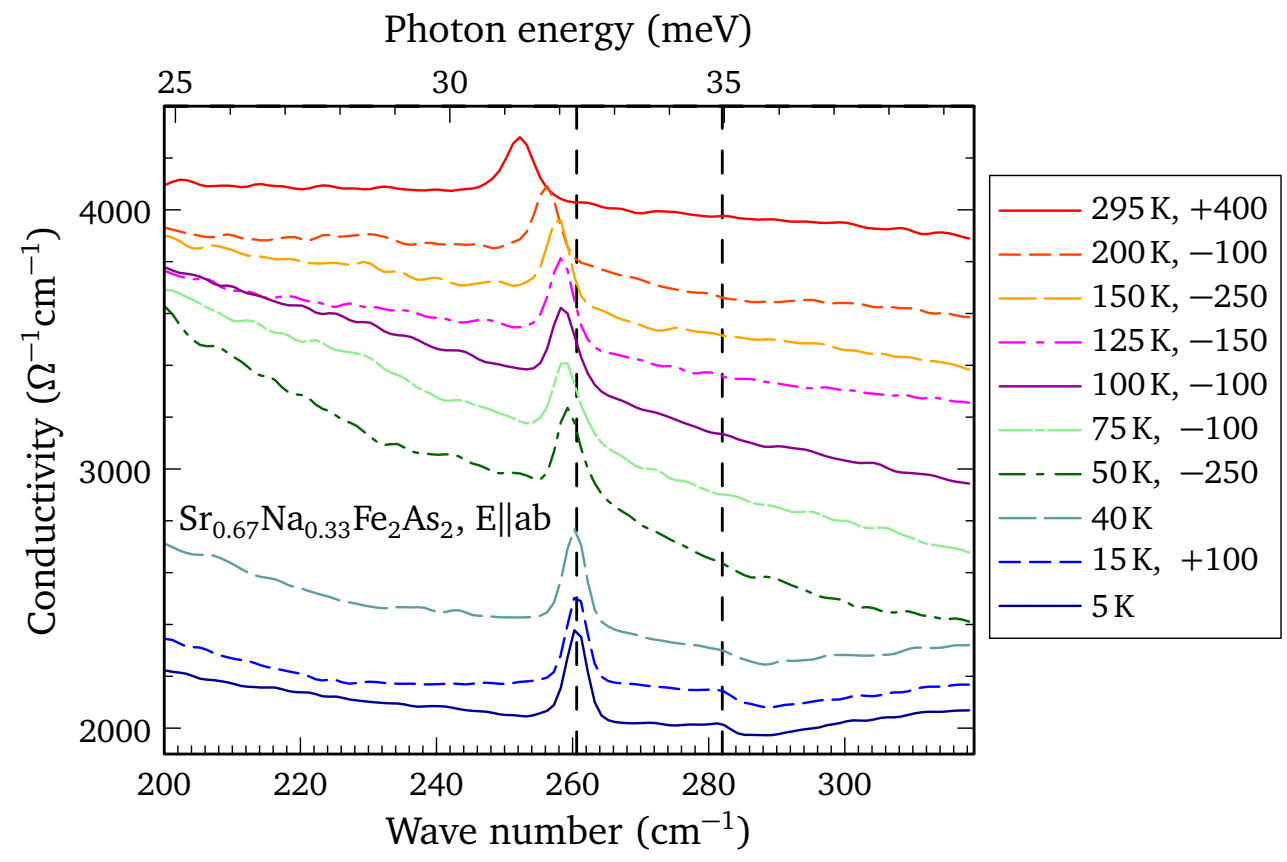

Figure S3. The temperature dependence of the real part of the optical conductivity of $\mathrm{Sr}_{0.67} \mathrm{Na}_{0.33} \mathrm{Fe}_{2} \mathrm{As}_{2}$ in the region of the in-plane infrared-active vibration at $\simeq 260 \mathrm{~cm}^{-1}$. The mode increases in frequency with decreasing temperature; while this mode does not split below $T_{N}$ in the orthorhombic phase, below $T_{r}$ a weak feature develops at $\simeq 282 \mathrm{~cm}^{-1}$. The number next to the temperature in the legend indicates the shift that has been applied to the conductivity data.

\section{PHONONS AND ZONE FOLDING}

The temperature dependence of the real part of the optical conductivity of $\mathrm{Sr}_{0.67} \mathrm{Na}_{0.33} \mathrm{Fe}_{2} \mathrm{As}_{2}$ for light polarized in the $\mathrm{Fe}-\mathrm{As}$ planes is shown in Fig. S3 in the region of the infrared-active vibration at $\simeq 260 \mathrm{~cm}^{-1}$. This mode increases in frequency with decreasing temperature, but it shows no evidence of either the weak $E_{u} \rightarrow B_{2 u}+B_{3 u}$ splitting or the anomalous increase in oscillator strength below $T_{N}$ that was observed in the parent compound [4]. However, below $T_{r}$ a weak satellite mode appears at $\simeq 282 \mathrm{~cm}^{-1}$. Similar behavior has been been observed in this mode in the $C_{4}$ phase of $\mathrm{Ba}_{1-x} \mathrm{~K}_{x} \mathrm{Fe}_{2} \mathrm{As}_{2}$ and has been attributed to Brillouin-zone folding [5]. Thus, while a tetragonal unit cell is recovered below $T_{r}$, the CSDW state results in the formation of a supercell that is considerably larger than the room-temperature tetragonal unit cell.

\section{ELECTRONIC STRUCTURE CALCULATIONS}

The electronic properties of $\mathrm{SrFe}_{2} \mathrm{As}_{2}$ for the non-magnetic high-temperature tetragonal $(I 4 / \mathrm{mmm})$ phase have been calculated using density functional theory (DFT) with the generalized gradient approximation (GGA) using the full-potential linearized augmented plane-wave (FP-LAPW) method [6] with local-orbital extensions [7] in the WIEN2k implementation [8]. An examination of different Monkhorst-Pack $k$-point meshes indicated that a $5 \times 5 \times 5$ $k$-point mesh with $R_{m t} k_{\max }=7.8$ was sufficient for good energy convergence. Beginning with the experimental unit cell [9], $a=3.927$, and $c=12.37 \AA$, with the atomic positions of Sr, Fe and As at $(0,0,0),\left(0, \frac{1}{2}, \frac{1}{4}\right)$, and $(0,0,0.361)$, respectively, the lattice parameters are adjusted and the total energy calculated for each structure in the absence of spin-orbit coupling (SOC); the lowest total energy in this phase space corresponds to the most stable geometry. The atomic fractional coordinates were then relaxed with respect to the total force, typically resulting in residual forces of less than $0.1 \mathrm{mRy} /$ a.u. per atom. This procedure was repeated until no further improvement was obtained, yielding $a=3.897$ and $c=12.27 \AA$; the position of the arsenic atom has shifted slightly to $(0,0,0.3528)$ [4]. The electronic band structure has been calculated with GGA in the absence of SOC for several different paths between high-symmetry points in the tetragonal unit cell, shown in Fig. S4(a) for the Fe $d_{x z} / d_{y z}$ orbital character; the effects SOC are shown in Fig. S4(b). This calculation accurately reproduces the results of earlier work [10], and clearly shows the presence of three hole-like bands at the center of the Brillouin zone, a well as two electron-like bands at the corner 

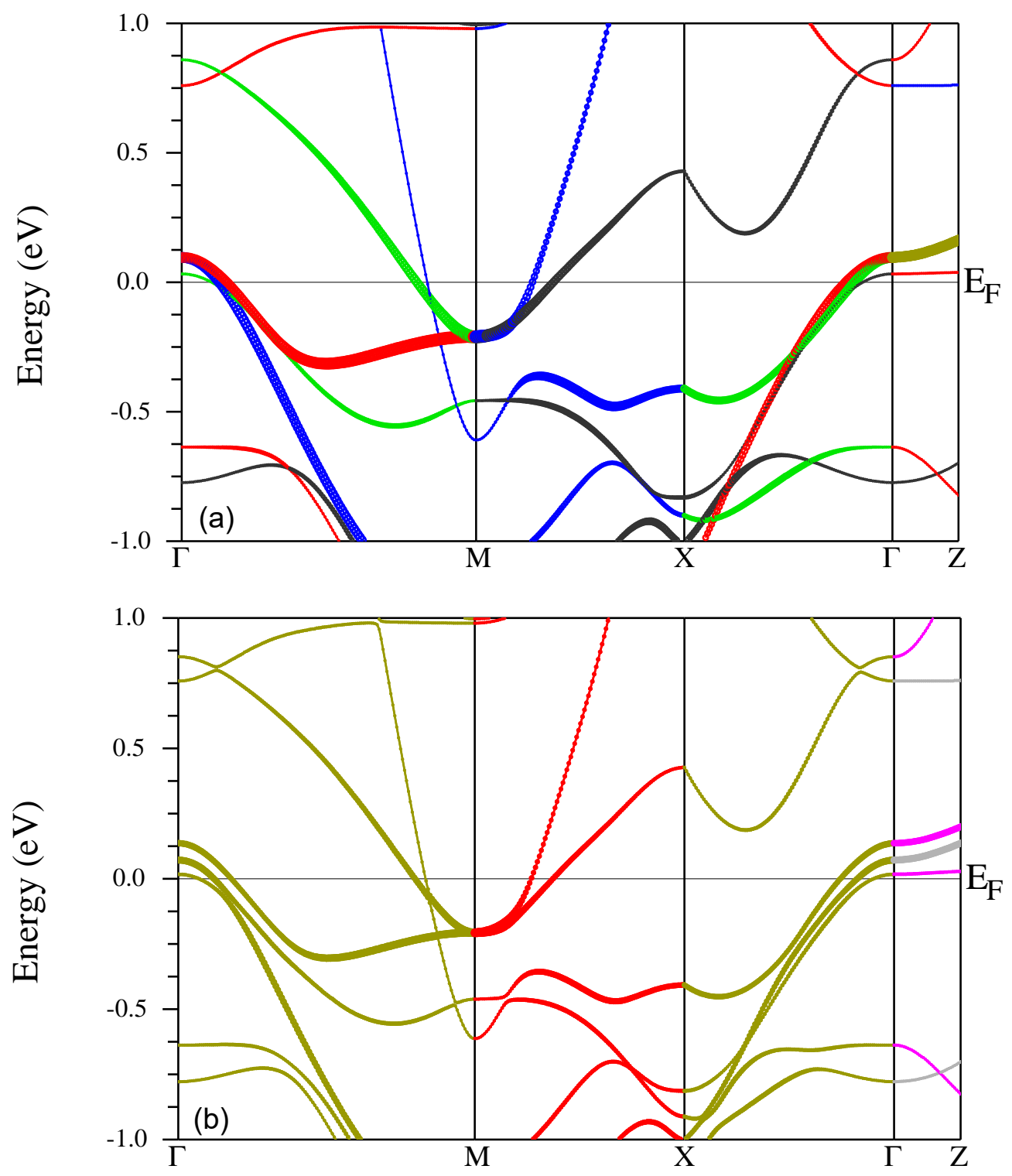

Figure S4. The calculated GGA electronic band structure of $\mathrm{SrFe}_{2} \mathrm{As}_{2}$ in the high-temperature tetragonal phase along several different paths between high symmetry points for the Fe $d_{x z} / d_{y z}$ orbital character plot (a) in the absence of SOC, and (b) with SOC.

(M) of the Brillouin zone.

\section{ANGLE RESOLVED PHOTOEMISSION}

Temperature dependent ARPES measurements have been performed to track the evolution of the electron and hole pockets in the various phases. Measurements at BNL, which focused on the electronic structure near the center of the Brillouin zone, were performed using $21.2 \mathrm{eV}$ light from a monochromator-filtered He I source (Omicron VUV5k) and a Scienta SES-R4000 electron spectrometer; emitted electrons were collected along the direction perpendicular to the light-surface mirror plane.

The Fermi surface of $\mathrm{Sr}_{0.67} \mathrm{Na}_{0.33} \mathrm{Fe}_{2} \mathrm{As}_{2}$, with the spectral weight integrated within a $\pm 10 \mathrm{meV}$ energy window with respect to the Fermi level, is shown below $T_{r}$ in the $C_{4}$ magnetic phase at $23 \mathrm{~K}$, in Fig. S5(a). Momentum cuts have been made along the $\left(k_{x}, k_{y}\right)$ directions around the anisotropic electron-like bands for the $\mathrm{M}$ points at $(-\pi,-\pi)$ and $(-\pi, \pi)$; because the material is twinned, these two points should be equivalent. The third cut samples 
(a)

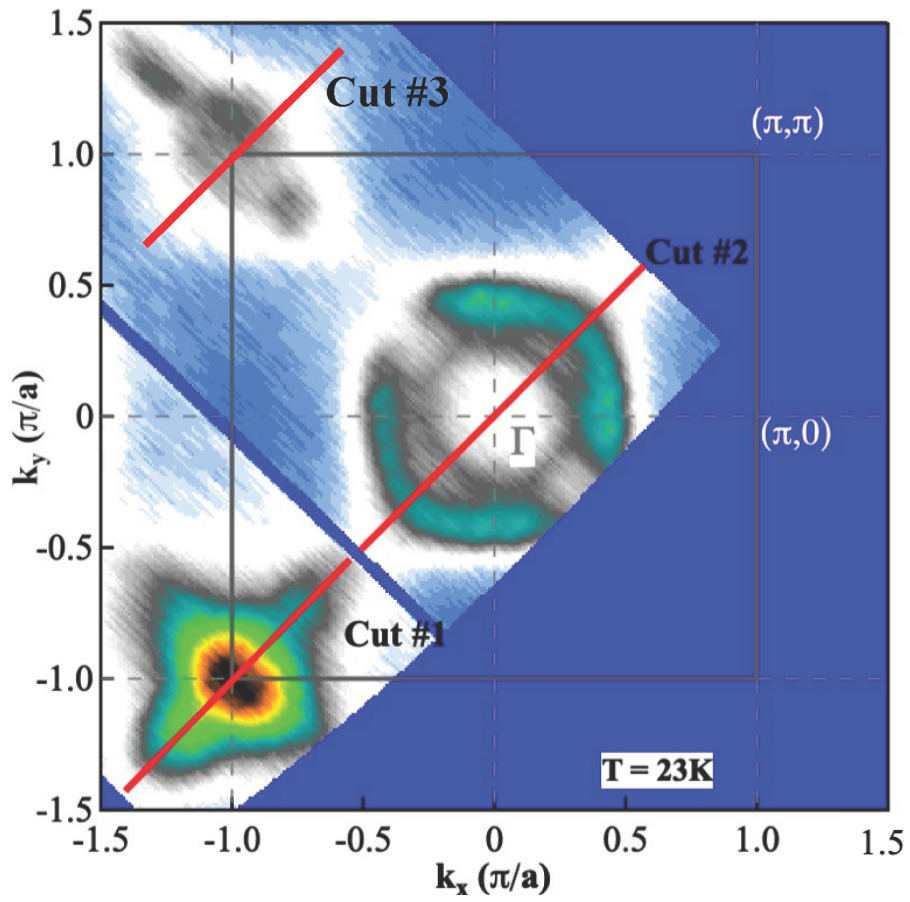

(b)

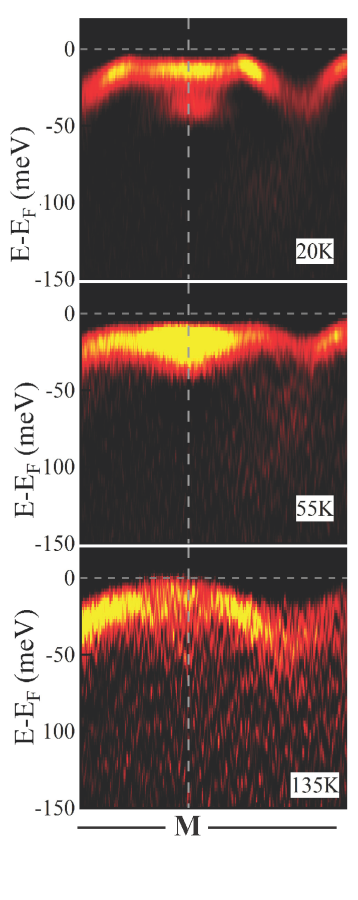

(c) Cut \#3@135 K

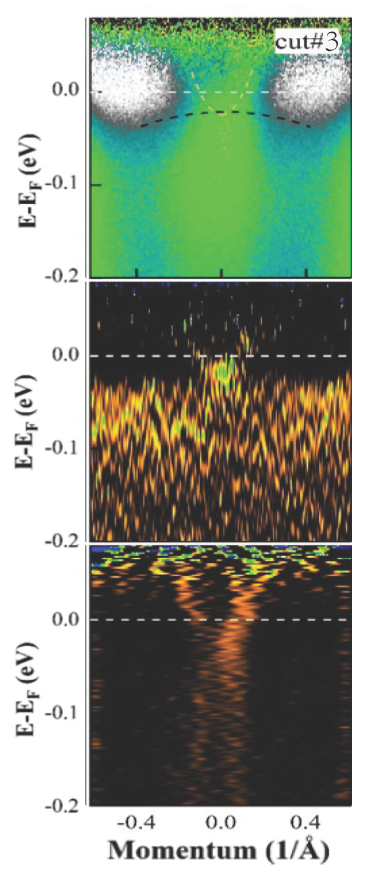

Figure S5. (a) Fermi surface mapping of $\mathrm{Sr}_{0.67} \mathrm{Na}_{0.33} \mathrm{Fe}_{2} \mathrm{As}_{2}$ in the $C_{4}$ magnetic phase at $23 \mathrm{~K}$ with the spectral weight integrated within a $\pm 10 \mathrm{meV}$ energy window with respect to the Fermi level, showing the hole-like pockets at the center $(\Gamma)$, and the electron-like pockets at the corner $(\mathrm{M})$ of the Brillouin zone. Several different cuts are shown along the $\Gamma \rightarrow \mathrm{M}$ path focus on the evolution of the hole and electron pockets. (b) The temperature dependence of the second derivative of the energy bands measured along the first cut around the $\mathrm{M}$ point at $(-\pi,-\pi)$ at $135 \mathrm{~K}\left(T>T_{\mathrm{N}}\right), 55 \mathrm{~K}\left(T_{r}<T<T_{\mathrm{N}}\right)$, and $20 \mathrm{~K}$ $\left(T_{c}<T<T_{r}\right)$. (c) The raw data for the $\mathrm{M}$ point at $(-\pi, \pi)$ at $135 \mathrm{~K}$ (upper panel); the second derivative of the energy distribution curves (middle); the momentum distribution curves (bottom).

the hole-like bands around the $\Gamma$ point. The temperature dependence of the second derivative of electron-like energy bands bands around the M point at $(-\pi,-\pi)$ are shown in Fig. S5(b). The The raw data for the M point at $(-\pi, \pi)$ at $135 \mathrm{~K}$ is shown in the upper panel of Fig. S5(c); the second derivative of the energy distribution curves (middle); the momentum distribution curves (bottom).

The results are shown at 18, 82 and $153 \mathrm{~K}$, for the iso-energy plots at $0,-20$, and $-40 \mathrm{meV}$, in Fig. S6. The second derivative of the iso-energy plots at 18, 82, and $153 \mathrm{~K}$ are shown in Fig. $\mathrm{S} 7$ at $-10,-20,-30$, and $-40 \mathrm{meV}$.

* Present address: Laboratorium für Festkörperphysik, ETH Zürich, CH-8093 Züich, Switzerland

$\dagger$ xgqiu@iphy.ac.cn

$\ddagger$ homes@bnl.gov

[1] Christopher C. Homes, M. Reedyk, D. A. Crandles, and T. Timusk, "Technique for measuring the reflectance of irregular, submillimeter-sized samples," Appl. Opt. 32, 2976-2983 (1993).

[2] M. Dressel and G. Grüner, Electrodynamics of Solids (Cambridge University Press, Cambridge, 2001).

[3] F. Wooten, Optical Properties of Solids (Academic Press, New York, 1972) pp. 244-250.

[4] C. C. Homes, Y. M. Dai, Ana Akrap, S. L. Bud'ko, and P. C. Canfield, "Vibrational anomalies in $A F e_{2} \mathrm{As}_{2}\left(A={ }^{2}\right.$ Ca, Sr, and Ba) single crystals," Phys. Rev. B 98, 035103 (2018).

[5] B. P. P. Mallett, Yu. G. Pashkevich, A. Gusev, Th. Wolf, and C. Bernhard, "Muon spin rotation study of the magnetic structure in the tetragonal antiferromagnetic state of weakly underdoped $\mathrm{Ba}_{1-x} \mathrm{~K}_{x} \mathrm{Fe}_{2} \mathrm{As}_{2}$," EPL (Europhysics Letters) 111, 57001 (2015).

[6] D. J. Singh, Planewaves, Pseudopotentials and the LAPW method (Kluwer Adademic, Boston, 1994).

[7] David Singh, "Ground-state properties of lanthanum: Treatment of extended-core states," Phys. Rev. B 43, 6388-6392 (1991).

[8] P. Blaha, K. Schwarz, G. K. H. Madsen, D. Kvasnicka and J. Luitz, WIEN2k, An augmented plane wave plus local orbitals 

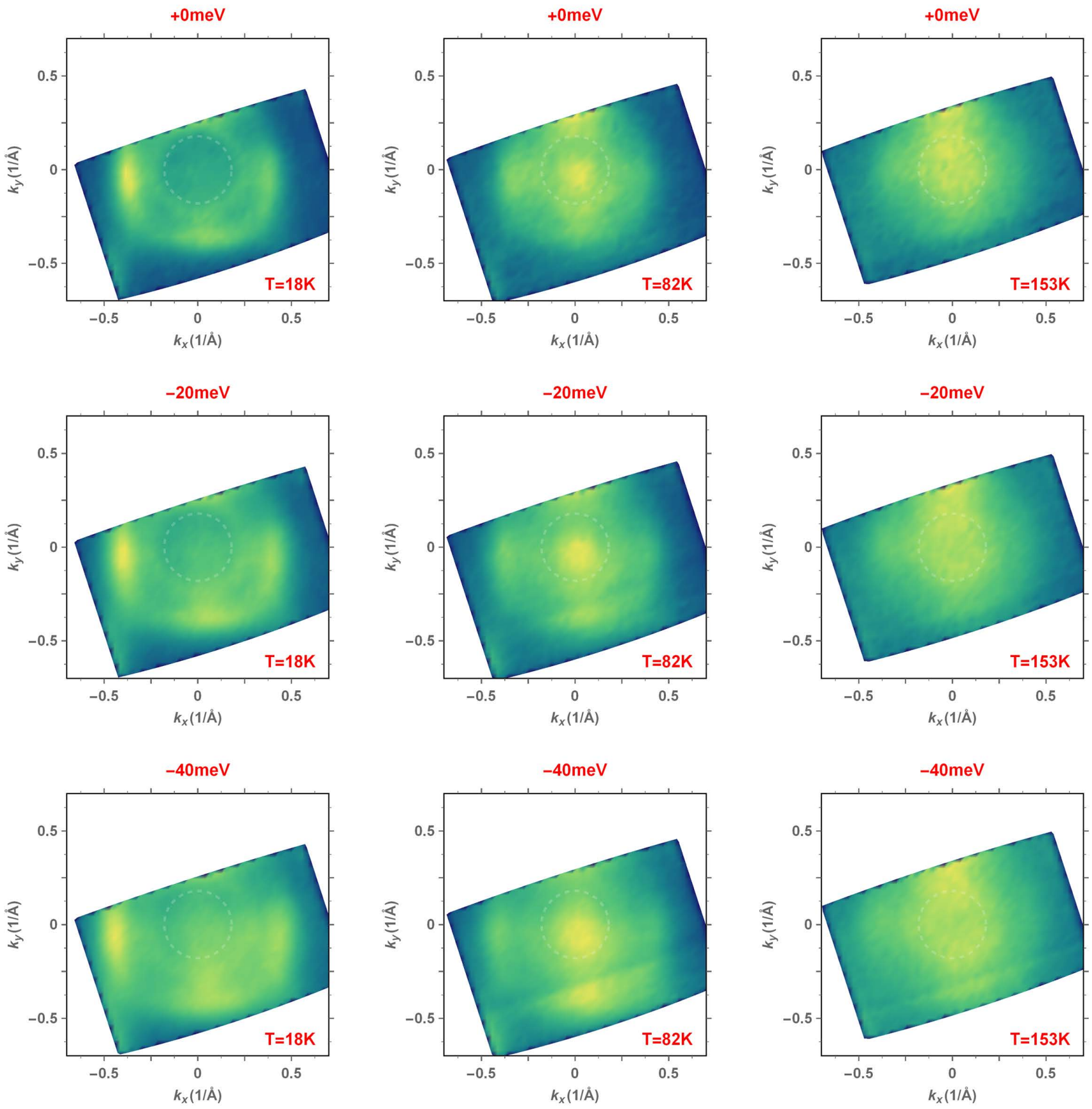

Figure S6. The angle resolved photoemission results for $\mathrm{Sr}_{0.67} \mathrm{Na}_{0.33} \mathrm{Fe}_{2} \mathrm{As}_{2}$ for the iso-energy ARPES spectra for three different temperatures, 18, 82 and $153 \mathrm{~K}$, at the Fermi level (first row), $-20 \mathrm{meV}$ (second row), and $-40 \mathrm{meV}$ (third row).

program for calculating crystal properties (Techn. Universität Wien, Austria, 2001).

[9] Marcus Tegel, Marianne Rotter, Veronika Wei $\beta$, Falko M Schappacher, Rainer Pöttgen, and Dirk Johrendt, "Structural and magnetic phase transitions in the ternary iron arsenides $\mathrm{SrFe}_{2} \mathrm{As}_{2}$ and $\mathrm{EuFe}_{2} \mathrm{As}_{2}$," J. Phys.: Condens. Matter 20, 452201 (2008).

[10] D. J. Singh, "Electronic structure and doping in $\mathrm{BaFe}_{2} \mathrm{As}_{2}$ and LiFeAs: Density functional calculations," Phys. Rev. B 78, 094511 (2008). 

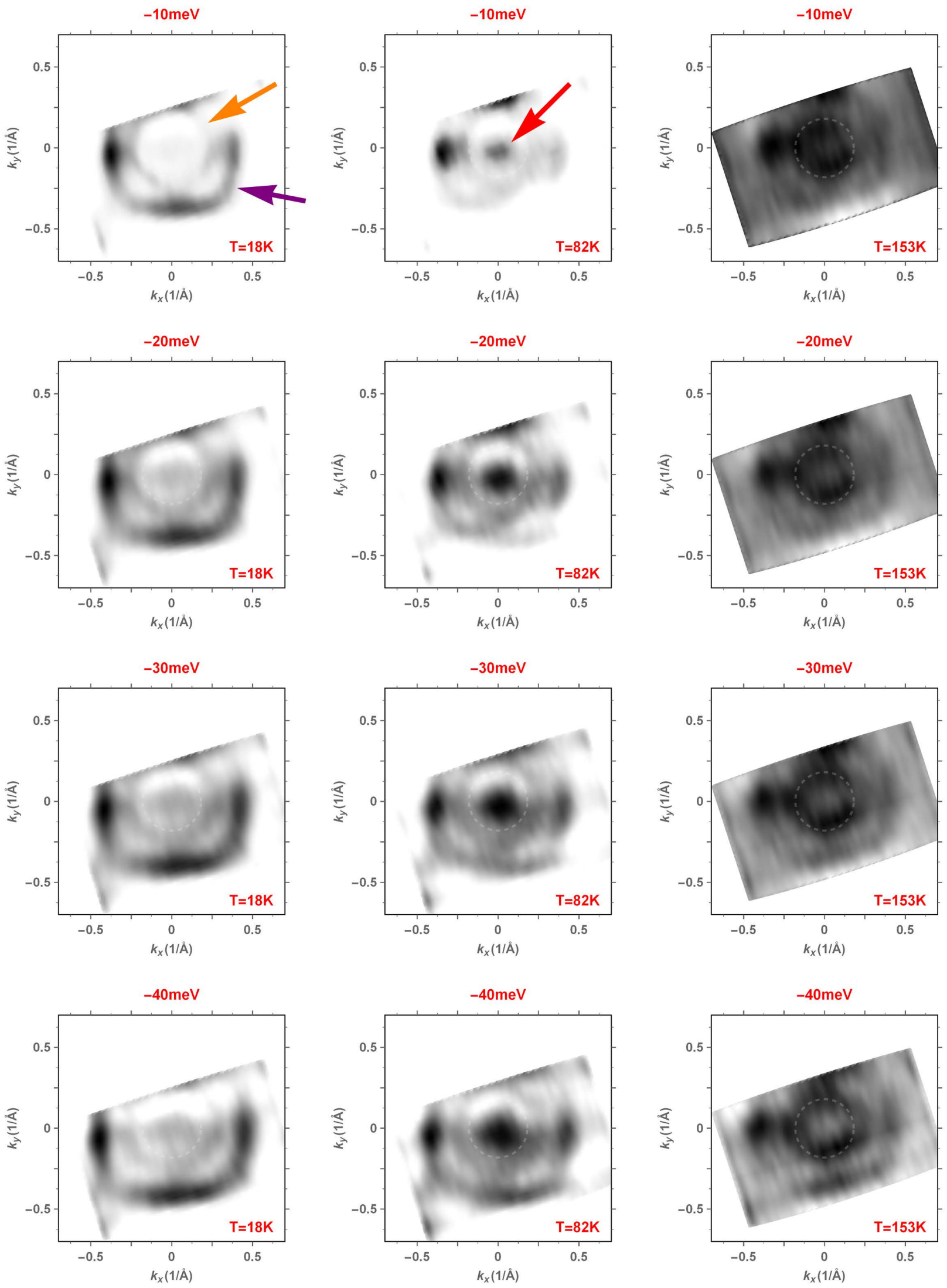

Figure S7. The angle resolved photoemission results for $\mathrm{Sr}_{0.67} \mathrm{Na}_{0.33} \mathrm{Fe}_{2} \mathrm{As}_{2}$ showing the second derivative of the iso-energy ARPES spectra at 18,82 , and $153 \mathrm{~K}$, at $-10 \mathrm{meV}$ (first row), $-20 \mathrm{meV}$ second row), $-30 \mathrm{meV}$ (third row), and $-40 \mathrm{meV}$ (last row). The colored arrows point to the corresponding colored parabolic bands in Fig. 6 of the main text. 